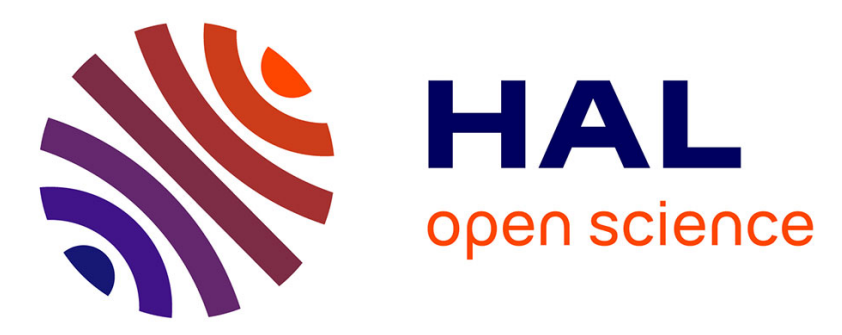

\title{
Algebraic-Closure-Based Moment Method for Unsteady Eulerian Simulations of Non-Isothermal Particle-Laden Turbulent Flows at Moderate Stokes Numbers in Dilute Regime
}

Enrica Masi, Olivier Simonin

\section{To cite this version:}

Enrica Masi, Olivier Simonin. Algebraic-Closure-Based Moment Method for Unsteady Eulerian Simulations of Non-Isothermal Particle-Laden Turbulent Flows at Moderate Stokes Numbers in Dilute Regime. Flow, Turbulence and Combustion, 2014, vol. 92 (n 1-2), pp. 121-145. 10.1007/s10494013-9516-2 . hal-01335782

\section{HAL Id: hal-01335782 \\ https://hal.science/hal-01335782}

Submitted on 22 Jun 2016

HAL is a multi-disciplinary open access archive for the deposit and dissemination of scientific research documents, whether they are published or not. The documents may come from teaching and research institutions in France or abroad, or from public or private research centers.
L'archive ouverte pluridisciplinaire HAL, est destinée au dépôt et à la diffusion de documents scientifiques de niveau recherche, publiés ou non, émanant des établissements d'enseignement et de recherche français ou étrangers, des laboratoires publics ou privés. 


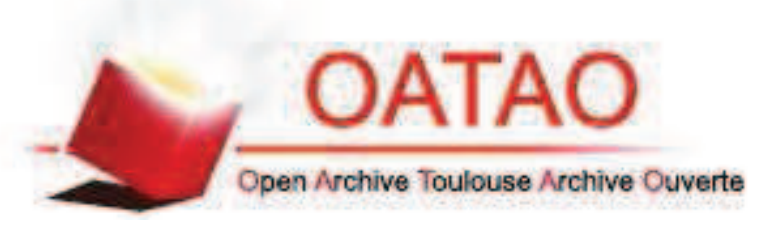

\section{Open Archive TOULOUSE Archive Ouverte (OATAO)}

OATAO is an open access repository that collects the work of Toulouse researchers and makes it freely available over the web where possible.

This is an author-deposited version published in : http://oatao.univ-toulouse.fr/ Eprints ID : 11775

To link to this article : DOI : 10.1007/s10494-013-9516-2

URL : http://dx.doi.org/10.1007/s10494-013-9516-2

To cite this version : Masi, Enrica and Simonin, Olivier AlgebraicClosure-Based Moment Method for Unsteady Eulerian Simulations of Non-Isothermal Particle-Laden Turbulent Flows at Moderate Stokes Numbers in Dilute Regime. (2014) Flow Turbulence and Combustion, vol. 92 (n 1-2). pp. 121-145. ISSN 1386-6184

Any correspondance concerning this service should be sent to the repository administrator: staff-oatao@,listes-diff.inp-toulouse.fr 


\title{
Algebraic-Closure-Based Moment Method for Unsteady Eulerian Simulations of Non-Isothermal Particle-Laden Turbulent Flows at Moderate Stokes Numbers in Dilute Regime
}

\author{
Enrica Masi • Olivier Simonin
}

\begin{abstract}
To model unsteady non-isothermal dilute particle-laden turbulent flows, an algebraic-closure-based moment method (ACBMM) is developed. ACBMM is a Eulerian approach for the dispersed phase conceived to be coupled with direct numerical simulations (DNSs) of the turbulence when an accurate local description of the turbulent mixture is required. It is based on the combination of a conditional probability density function (PDF) approach, which provides local instantaneous Eulerian equations for the low-order moments of the PDF, and appropriate constitutive relations, as algebraic closures, which are necessary to close the set of conservation equations. The computed low-order moments are the mesoscopic particle number density, particle velocity and particle temperature and the unclosed higherorder moments are the particle random uncorrelated motion (RUM) stress tensor and the RUM heat flux (RUM-HF) which appear in the particle momentum and enthalpy equations, respectively. The RUM stress tensor is closed by an additional transport equation for the trace of the tensor and a polynomial representation for tensor functions modeling its deviatoric part. The polynomial representation is used in the framework of an assumption of equilibrium of the RUM anisotropy and leads to an explicit algebraic stress model (2థEASM). Similarly, the RUM-HF is modeled assuming equilibrium of the scaled heat flux and explicit self-consistent solutions (2ФEAHFM) are found by analogy with turbulent heat flux models. As $2 \Phi E A H F M$ entails the computation of the RUM temperature variance, an additional transport equation is developed for it. By means of an a priori analysis, the algebraic closures
\end{abstract}

E. Masi ( $\bowtie) \cdot$ O. Simonin INPT-UPS, IMFT, Allée Camille Soula, Université de Toulouse, 31400 Toulouse, France e-mail: emasi@imft.fr 
developed by the present study are assessed against actual particle Eulerian fields which are extracted from particle Lagrangian simulations coupled with DNS of a temporal non-isothermal particle-laden turbulent planar jet, for various Stokes numbers. Results show that both 2ФEASM and 2ФEAHFM are successful in reproducing the unclosed moments up to moderate turbulent-macroscale Stokes numbers allowing the ACBMM to accurately predict the unsteady non-isothermal dispersed phase.

Keywords Unsteady Eulerian two-fluid model $\cdot$ Particle-laden flows $\cdot$ DNS

\section{Introduction}

Due to the vast importance that particle-laden turbulent flows have nowadays in many industrial applications, providing for a modeling which is both accurate and computationally affordable represents an important challenge. The main constraint arises from the need to have an accurate local description of the turbulent mixture, for example in unsteady turbulent flows or complex geometry, which involves very high computational costs, sometimes prohibitive for real applications. At industrial scale, a turbulence which needs highly-accurate solutions is modeled by means of the large-eddy simulation (LES). The LES approach relies on the filtered directnumerical-simulation (DNS) equations which are closed by modeling the unresolved subgrid-scale terms. Then, the evolution of the properties of particles which are coupled with LES (or DNS) of turbulent flows may be obtained by either a Lagrangian or a Eulerian approach. The former is an uncontroversial accurate method, in dilute flows, when used for particle smaller than the Kolmogorov lengthscale and when coupled with DNS of turbulence, however it is unrealistic at industrial scale where a huge number of particles has to be used. The latter is instead much more competitive in terms of computational costs and it may represent a valid alternative to the former provided that the same level of accuracy is ensured. This method is referred to as Eulerian-Eulerian DNS (or LES) approach. In the Eulerian-Eulerian approach, the particles are described as a continuum and the two-phase governing equations are solved separately and coupled through interphase exchange terms. In this study we will focus on the Eulerian-Eulerian DNS modeling as the baseline of the Eulerian-Eulerian LES approach [39]. The aim of this work is indeed to provide an unsteady two-fluid model appropriate for DNS of very dilute particle-laden flows, in which a local prediction of the particle behavior is mandatory. The proposed model should not be confused with the two-fluid models available in the literature which are modeling the fluid turbulence in the frame of the Reynolds-averaged NavierStokes (RANS) approach or dedicated to dense particulate flow regimes [see, e.g., $1,6,13,46,54,69,70]$.

\subsection{Unsteady Eulerian particle models}

In the literature, unsteady Eulerian models for particles have been proposed but they are limited to small Stokes numbers $\left(S t_{K}<1\right.$ based on the Kolmogorov timescale, $S t<<1$ based on a characteristic large timescale of the turbulence seen by the particles. Hereafter, we will refer to $S t$ as turbulent-macroscale Stokes number). 
We recall the fast (or equilibrium) Eulerian method of Maxey [35], Ferry and Balachandar $[14,15]$, based on a Taylor expansion of the particle velocity equation, the multi-fluid approach of Laurent and Massot [27], which assumes a monokinetic description of the particle velocity, and the two-fluid model of Druzhinin and Elghobashi [11, 12] which is based on a spatial average of the particle equations. Successfully assessed for small particle inertia, these approaches fail when the Stokes number, $S t_{K}$, approaches unity $[44,51]$. The main reason must be sought in the failure of the assumption of the uniqueness of the particle (velocity and temperature) distributions as the Stokes number increases. Recently, Simonin et al. [56] and Février et al. [16] have shown that in order to be able to model the dispersed phase corresponding to large Stokes numbers, a Eulerian approach should account for the effect of the crossing between particle trajectories. This phenomenon has a great impact on the thermal characterization of the dispersed phase as well. Indeed, the occurrence of crossing trajectories entails that particles convey information of their interactions with very distant and independent turbulent eddies, that is with different dynamic and thermal turbulent scales. This effect involves many different velocities and temperatures in the same volume of control, violating the assumption of the uniqueness of the particle velocity and temperature distributions. An extension of the statistical approach accounting for non-isothermal conditions may be found in [33]. Particle velocity and temperature are partitioned in two contributions: i) a Eulerian particle (velocity and temperature) field, referred to as mesoscopic field, which is spatially correlated and shared by all the particles and which accounts for correlations between particles and between particles and fluid; ii) a spatiallyuncorrelated particle (velocity and temperature) contribution, referred to as random uncorrelated motion (RUM) contribution, associated to each particle and resulting from the chaotic particles' behavior. The RUM contribution is characterized in terms of Eulerian fields of particle velocity and temperature moments; the larger is the particle inertia the more important is the RUM. The existence of a spatiallyuncorrelated contribution and its connection with singularities and caustic formation is a timely topic of research [22, 24, 37].

\subsection{The statistical approach and the question of the closures}

The model proposed by Simonin et al. [56] and Février et al. [16], and referred to as Mesoscopic Eulerian Formalism (MEF), allows to define a conditional oneparticle probability density function (PDF) which, in dilute regime, where particle interactions may be neglected, provides a complete description of the dispersed phase. By analogy with the kinetic theory of gases, the conditional-PDF evolutions follow a Boltzmann-like equation which is closed at the same level than the Lagrangian equations governing the discrete particle variables. From the solution of the integro-differential PDF kinetic equation, one can obtain the moments of the PDF which provide a full description of the dispersed phase in a Eulerian framework. However, a direct solution of the kinetic equation is too expensive because of the large number of dimensions appearing in the phase space. For this reason, a moment method relaying on the solution of the differential equations of the PDF moments is somewhat preferable. Unfortunately, a closed kinetic equation does not address the closure problem in real space since its evolution in the phase space creates an infinite set of coupled moment equations in real space. Therefore, any 
finite set of moment equations has to be supplemented by closure models of the unknown moments written in terms of the computed ones. In very dilute regime, in which the system formed by particles (rather than molecules) may be considered very far from equilibrium, closures for the particulate phase can be provided by analogy with the kinetic theory of rarefied gases using a high-order Chapman-Enskog expansion [5] of the kinetic equation or a Grad's approach [21]. According to the Grad's approximation, the stress tensor and the heat flux are not treated as auxiliary variables but solved according to their own conservation equations as variables of state. This approach, which uses an expansion of the PDF in terms of Hermite polynomials, has the same order in powers of Knudsen number as the Burnett equation [60] which is derived by a second-order Chapman-Enskog expansion of the kinetic equation. A third-order approximation of the Grad's approach, having the same order as a super-Burnett approximation, was recently proposed by Struchtrup and Torrilhon [59]. The Grad's approach turns out very similar to some kineticbased-moment methods (KBMMs) recently proposed for the particulate phase [63] which use an anisotropic Gaussian particle velocity distribution and define a set of conservation equations where the third-order correlation is closed according to the Gaussian assumption. On the basis of kinetic and mathematical arguments, higher-order closures given by high-order-moment transport equations are instead provided by the quadrature-based-moment methods (QBMMs); the goal of QBMMs is to model the dispersed phase when the Stokes number considerably increases and a large number of moments is required [8, 9, 17, 18, 25, 28, 29, 34, 40]. These high-order-moment methods are based on a presumed particle property distribution which may be modeled as a sum of Dirac delta functions, in order to account for the effect of multiple crossing trajectories [67], or by a multi-Gaussian distribution $[3,62]$. The latter has been proposed to overcome the problem of the singularities which may be generated by unphysical delta shocks when a sum of Dirac delta functions is used for solving multiple crossing with a limited number of quadrature points [4]. These approaches using third, fourth and even higher order moments for modeling the dispersed phase at large/very large Stokes numbers $(S t>>1)$, even if accurate are in fact too expensive to be used in CFD codes and unrealistic for simulating industrial configurations. For moderate Stokes numbers $(S t>1)$, a moment method using constitutive relations for closing the second-order moments instead of conservation equations may be much more effective. For non-equilibrium fluids, non-linear second-order constitutive relations for the stress tensor and the heat flux were developed by Wong et al. [66] using the correlation function theory, and they correspond to the order of the Burnett approximation in kinetic theory of gases. Chen et al. [7] (among other authors) provided an analytical expression using a Chapman-Enskog expansion of the Boltzmann equation. They showed that the firstorder approximation leads to the well-known constitutive relation for Newtonian fluids, while the second-order approximation leads to a non-Newtonian expression in which the stresses are related to a memory-effect term, represented by the Lagrangian derivative of the strain, and to nonlinear tensorial terms. On the basis of the fact that there exist various orders of approximation based on the choice of the highest-order moment conservation equation to be retained, and that to any order of approximation only a part of the exact solution of the kinetic equation is provided, the question is then whether is possible, by using accurate second-order 
approximation closures, to reduce the set of conservation equations to the low-order moments of the PDF (i.e., particle density, velocity and temperature) and for which range of Stokes numbers this may apply.

Models for the dispersed phase which are not based on a presumed PDF in the phase space but which use algebraic closures in physical space are here referred to as algebraic-closure-based moment methods (ACBMMs). ACBMMs using secondorder moment equations and modeling the third-order correlation by using algebraic closures based on physical arguments were developed by Simonin [55], He and Simonin [23], Sakiz and Simonin [49]. Used in the frame of the RANS approach, they can easily be extended to a DNS/LES framework by using the one-particle conditional PDF approach for defining the moments of the dispersed-phase at the mesoscale. An ACBMM solving the low-order moments of the dispersed phase (the mesoscopic particle number density and velocity) and modeling the second-order moment appearing in particle momentum equation, namely the RUM particle kinetic stress tensor (RUM-ST), has been proposed by Simonin et al. [56] and Kaufmann et al. [26]. The RUM-ST is closed by an additional transport equation for the trace of the tensor and a viscosity assumption is used for its deviatoric part. In nonisothermal conditions, Masi et al. [31] introduced an equation for the mesoscopic particle temperature and suggested to close the second-order moment appearing in the enthalpy equation (the RUM heat flux (RUM-HF)) by means of an eddydiffusivity assumption. Unfortunately, a recent work [30] has shown that these linear algebraic closures are doomed to fail in unsteady turbulent mean sheared flows as the Stokes number increases. In isothermal conditions, such a failure was already pointed out by Riber [47] when performing preliminary Eulerian-Eulerian simulations of a particle-laden turbulent jet confined in a pipe flow. On the basis of the analogy with the kinetic theory of rarefied gases, it is clear that higher-order constitutive relations should be provided in order to hope to capture, with a sufficient level of accuracy, the particles' behavior in dilute regime and high particle inertia. By this work, second-order non-linear closures for the RUM-ST and the RUM-HF are proposed. They enable the ACBMM approach to accurately predict non-isothermal unsteady particle-laden turbulent flows in the presence of mean shear at moderate Stokes number as well. This modeling is a part of a Ph.D. work [30]. Results of preliminary isothermal and non-isothermal Eulerian-Eulerian DNS using the models developed in [30] may be found in [10,52].

The manuscript is organized as follows. In Section 2, the point-particle Lagrangian method and the unsteady Eulerian statistical approach are presented. The former is used to perform deterministic simulations of reference and building a Eulerian database which will be used for assessing, by an a priori analysis, the closures developed by the present study. The Eulerian statistical approach is used to develop a set of unclosed conservation equations which are proposed for the modeling of the non-isothermal dispersed phase in a DNS framework. Closures for such equations, i.e. for the RUM-ST and the RUM-HF, are developed and described in Sections 3 and 4, respectively. Such closures are a priori assessed against actual particle Eulerian fields which are extracted from particle Lagrangian simulations coupled with DNSs of a temporal non-isothermal particle-laden turbulent planar jet, for various Stokes numbers. Results of the assessment are presented in Section 5. Summary and conclusions are given in Section 6. 


\section{Lagrangian Versus Eulerian Conditional Statistical Approach}

\subsection{Lagrangian approach for non-isothermal particulate phase}

Using the Lagrangian technique, the behavior of particles interacting with nonisothermal turbulent flows is investigated by the integration in time of the Newton equations of the particle position, velocity and temperature. When the mixture is composed of spherical, rigid, non-rotating and non-interacting heavy particles with a diameter smaller than the Kolmogorov lengthscale and an infinite internal conductivity, equations are strongly simplified since many contributions may be neglected. Assuming neither gravity nor radiative transfer, the equations governing the motion and the heat exchanges of each particle are $[36,68]$

$$
\frac{\mathrm{d} \mathbf{x}_{p}}{\mathrm{~d} t}=\boldsymbol{v}_{p}, \quad \frac{\mathrm{d} \boldsymbol{v}_{p}}{\mathrm{~d} t}=-\frac{1}{\tau_{p}}\left(\boldsymbol{v}_{p}-\mathbf{u}_{f @ p}\right), \quad \frac{\mathrm{d} T_{p}}{\mathrm{~d} t}=-\frac{1}{\tau_{\theta}}\left(T_{p}-T_{f @ p}\right)
$$

where $\mathbf{u}_{f @ p}(t)=\mathbf{u}_{f}\left(\mathbf{x}_{p}(t), t\right)$ and $T_{f @ p}(t)=T_{f}\left(\mathbf{x}_{p}(t), t\right)$ are the undisturbed fluid velocity and temperature at the particle center location and $\tau_{p}$ and $\tau_{\theta}$ are the dynamic and thermal particle response times, respectively. The general expressions for the response times accounting for non-linearities of external forces and heat exchanges reads

$$
\tau_{p}=\frac{4 \rho_{p} d_{p}}{3 \rho_{f} C_{D}\left\|\boldsymbol{v}_{p}-\mathbf{u}_{f @ p}\right\|}, \quad \tau_{\theta}=\frac{\operatorname{Pr} \rho_{p} d_{p}^{2} C_{p p}}{6 \mathrm{Nu} \mu_{f} C_{p f}}
$$

where $C_{D}$ and $\mathrm{Nu}$ are the corrected drag coefficient and Nusselt number $[45,50]$

$$
C_{D}=\frac{24}{\operatorname{Re}_{p}}\left(1+0.15 \operatorname{Re}_{p}^{0.687}\right), \quad \mathrm{Nu}=2+0.55 \operatorname{Re}_{p}^{1 / 2} \operatorname{Pr}^{1 / 3},
$$

formulated in terms of the particle Reynolds number which is defined as $\operatorname{Re}_{p}=$ $\left\|\boldsymbol{v}_{p}-\mathbf{u}_{f @ p}\right\| d_{p} / v_{f}$. In the above equations, Pr is the Prandtl number chosen smaller than unity in order to ensure well-resolved thermal small scales, $\mu_{f}$ and $v_{f}$ are the dynamic and kinematic fluid viscosity respectively, $\rho_{f}$ is the density of the fluid, $\rho_{p}$ and $d_{p}$ are the particle density and the particle diameter, and $\alpha=C_{p p} / C_{p f}$ is the particle-to-fluid heat capacity ratio. In the Stokes regime, the two times may be provided by the simplified expressions

$$
\tau_{p}=\frac{\rho_{p} d_{p}^{2}}{18 \mu_{f}}, \quad \tau_{\theta}=\frac{\operatorname{Pr} \rho_{p} d_{p}^{2} C_{p p}}{12 \mu_{f} C_{p f}}=\frac{3}{2} \operatorname{Pr} \alpha \tau_{p} .
$$

The system of Eq. 1 is coupled with an evolving turbulent flow which is exactly resolved by using a DNS approach.

\subsection{Eulerian conditional statistical approach for non-isothermal dispersed phase}

A detailed description of the conditional statistical approach is given in [16]. Its extension to non-isothermal conditions is detailed in [33]. The only difference between [33] and the present study is that here the particle mass is considered as a constant. The one-particle PDF, $\tilde{f}_{p}\left(\boldsymbol{x}, \boldsymbol{c}_{p}, \xi_{p}, t ; \mathscr{H}_{f}\right)$, conditional on a given fluid flow realization $\mathscr{H}_{f}$, is therefore defined in the phase space as a function of the only 
particle translation velocity $\boldsymbol{c}_{p}$ and particle temperature $\xi_{p}$, other than of the position $\mathbf{x}$ and of the time $t$. The mesoscopic ensemble average of any Lagrangian quantity $\mathbf{g}_{p}(t)$ which can be written as a function of the particle velocity and temperature, $\mathbf{g}_{p}(t)=\boldsymbol{\gamma}\left(\boldsymbol{u}_{p}(t), T_{p}(t)\right)$, is obtained by integration over the particle space and leads to the mesoscopic field

$$
\tilde{\mathbf{g}}_{p}(\boldsymbol{x}, t)=\frac{1}{\tilde{n}_{p}(\boldsymbol{x}, t)} \int \boldsymbol{\gamma}\left(\boldsymbol{c}_{p}, \xi_{p}\right) \tilde{f}_{p}\left(\boldsymbol{x}, \boldsymbol{c}_{p}, \xi_{p}, t ; \mathscr{H}_{f}\right) d \boldsymbol{c}_{p} d \xi_{p}
$$

where $\tilde{n}_{p}$ is the mesoscopic particle number density obtained by the integration of $\tilde{f}_{p}$. Hereinafter, for the sake of synthesis, the contracted notation $\tilde{\mathbf{g}}_{p}(\boldsymbol{x}, t)=$ $<\mathbf{g}_{p}(t) \mid \boldsymbol{x}_{p}(t)=\boldsymbol{x} ; \mathscr{H}_{f}>$ is used. According to the MEF, instantaneous velocity and temperature of each particle may be decomposed in two contributions, an instantaneous Eulerian field and a residual particle component

$$
u_{p, i}(t)=\widetilde{u}_{p, i}\left(\boldsymbol{x}_{p}(t), t\right)+\delta u_{p, i}(t), \quad T_{p}(t)=\widetilde{T}_{p}\left(\boldsymbol{x}_{p}(t), t\right)+\delta T_{p}(t) .
$$

The first contribution is a correlated continuous field shared by all the particles and written in an Eulerian framework, it leads to the first-order moments of the conditional PDF, namely the mesoscopic particle velocity and the mesoscopic particle temperature

$$
\widetilde{u}_{p, i}(\boldsymbol{x}, t)=<u_{p, i}(t)\left|\boldsymbol{x}_{p}(t)=\boldsymbol{x} ; \mathscr{H}_{f}>, \widetilde{T}_{p}(\boldsymbol{x}, t)=<T_{p}(t)\right| \boldsymbol{x}_{p}(t)=\boldsymbol{x} ; \mathscr{H}_{f}>;
$$

the second contribution is a random spatially-uncorrelated component, associated with each particle and defined along the particle path. It stems from the chaotic motion of the particles and it is characterized in terms of Eulerian fields of particle velocity and temperature higher central moments. Second-order moments of the conditional PDF are the RUM particle kinetic stress tensor

$$
\delta R_{p, i j}(\boldsymbol{x}, t)=\left\langle\delta u_{p, i}(t) \delta u_{p, j}(t) \mid \boldsymbol{x}_{p}(t)=\boldsymbol{x} ; \mathscr{H}_{f}\right\rangle,
$$

whose trace is twice the RUM particle kinetic energy $2 \delta \theta_{p}=\delta R_{p, i i}$. The RUM particle temperature variance

$$
\delta \theta_{\theta}(\boldsymbol{x}, t)=\frac{1}{2}\left\langle\delta T_{p}(t) \delta T_{p}(t) \mid \boldsymbol{x}_{p}(t)=\boldsymbol{x} ; \mathscr{H}_{f}\right\rangle,
$$

and the RUM-HF

$$
C_{p p} \delta \Theta_{p, i}(\boldsymbol{x}, t)=C_{p p}\left\langle\delta v_{p, i}(t) \delta T_{p}(t) \mid \boldsymbol{x}_{p}(t)=\boldsymbol{x} ; \mathscr{H}_{f}\right\rangle^{1} .
$$

Third-order moments of the RUM are the third-order RUM velocity correlation

$$
\delta Q_{p, i j k}(\boldsymbol{x}, t)=\left\langle\delta u_{p, i}(t) \delta u_{p, j}(t) \delta u_{p, k}(t) \mid \boldsymbol{x}_{p}(t)=\boldsymbol{x} ; \mathscr{H}_{f}\right\rangle,
$$

and the third-order RUM velocity with temperature correlations

$$
\begin{aligned}
\delta \Omega_{p, i}(\boldsymbol{x}, t) & =\left\langle\delta v_{p, i}(t) \delta T_{p}(t) \delta T_{p}(t) \mid \boldsymbol{x}_{p}(t)=\boldsymbol{x} ; \mathscr{H}_{f}\right\rangle, \\
\delta \Delta_{p, i j}(\boldsymbol{x}, t) & =\left\langle\delta v_{p, i}(t) \delta v_{p, j}(t) \delta T_{p}(t) \mid \boldsymbol{x}_{p}(t)=\boldsymbol{x} ; \mathscr{H}_{f}\right\rangle .
\end{aligned}
$$

\footnotetext{
${ }^{1}$ The particle specific heat capacity $C_{p p}$ was intentionally pulled out of the conditional mean operator as we assume it is constant. For that reason, it will be withdrawn from the enthalpy equation and hereinafter, for the sake of simplicity, we will refer to the quantity $\delta \Theta_{p, i}(\boldsymbol{x}, t)$ as the RUM heat flux even if it does not have units of heat flux without accounting for $C_{p p}$ and the particle density as well.
} 
In the framework of the PDF approach, the moment transport equations are obtained by analogy with the kinetic theory of gases [5]. Without gravity, if the particle diameter is smaller or equal than the Kolmogorov lengthscale and the particle-tofluid density ratio is large, only the drag force modifies the PDF. Further, assuming stationary inter-phase heat transfers and no radiant sources, the equation of the PDF is written as

$$
\begin{aligned}
\frac{\partial}{\partial t} \tilde{f}_{p}^{(1)}+\frac{\partial}{\partial x_{j}}\left[c_{p, j} \tilde{f}_{p}^{(1)}\right]= & +\frac{\partial}{\partial c_{p, j}}\left[\frac{\left(c_{p, j}-u_{f @ p, j}\right)}{\tau_{p}} \tilde{f}_{p}^{(1)}\right] \\
& +\frac{\partial}{\partial \xi_{p}}\left[\frac{\left(\xi_{p}-T_{f @ p}\right)}{\tau_{\theta}} \tilde{f}_{p}^{(1)}\right]+\left(\frac{\partial \tilde{f}_{p}^{(1)}}{\partial t}\right)_{\text {coll }} .
\end{aligned}
$$

The first and the second terms on the right hand side (r.h.s.) account for the effect of external forces and heat exchanges acting on the particle respectively; they are closed according to the same assumptions used for closing the Lagrangian particle equation [see, e.g. 68]. The last term in Eq. 14 accounts for the modification in the distribution function due to the particle interactions (collisions, coalescence). In very dilute regimes, the latter may be neglected provided that the typical collision time is much greater than the particle response time. Nevertheless, the formalism may theoretically include inter-particle collisions assuming that they do not directly induce spatial correlations in the particle velocity distribution. Local and instantaneous Eulerian equations are then obtained from Eq. 14 by multiplying by any function $\boldsymbol{\gamma}\left(\boldsymbol{c}_{p}, \xi_{p}\right)$ and integrating over the particle velocity-temperature space. At the first order, neglecting collisions, the local and instantaneous isothermal dispersed phase is described by the evolution of the mesoscopic particle number density and velocity [56]

$$
\begin{gathered}
\frac{\partial \tilde{n}_{p}}{\partial t}+\frac{\partial \tilde{n}_{p} \widetilde{u}_{p, i}}{\partial x_{i}}=0 \\
\frac{\partial \tilde{n}_{p} \widetilde{u}_{p, i}}{\partial t}+\frac{\partial \tilde{n}_{p} \widetilde{u}_{p, i} \widetilde{u}_{p, j}}{\partial x_{j}}=-\frac{\tilde{n}_{p}}{\tilde{\tau}_{p}}\left(\widetilde{u}_{p, i}-u_{f, i}\right)-\frac{\partial \tilde{n}_{p} \delta R_{p, i j}}{\partial x_{j}} ;
\end{gathered}
$$

in non-isothermal conditions an equation for the mesoscopic temperature should be accounted for

$$
\frac{\partial \tilde{n}_{p} \widetilde{T}_{p}}{\partial t}+\frac{\partial \tilde{n}_{p} \tilde{u}_{p, j} \widetilde{T}_{p}}{\partial x_{j}}=-\frac{\tilde{n}_{p}}{\tilde{\tau}_{\theta}}\left(\widetilde{T}_{p}-T_{f}\right)-\frac{\partial \tilde{n}_{p} \delta \Theta_{p, j}}{\partial x_{j}}
$$

In the above Eqs. $\tilde{\tau}_{p}=<1 / \tau_{p} \mid \mathscr{H}_{f}>^{-1}$ and $\tilde{\tau}_{\theta}=<1 / \tau_{\theta} \mid \mathscr{H}_{f}>^{-1}$ are the mesoscopic dynamic and thermal response times. The first term on the r.h.s. of Eq. 16 accounts for the effects of the drag force and the second one is the transport due to the RUM second-order moment. Into Eq. 17, the first r.h.s. contribution represents the interphase heat exchanges and the second one is the transport of the mesoscopic 
temperature due to the RUM heat flux. At the second order, the statistical modeling leads to an equation for the particle kinetic RUM-ST [38]

$$
\begin{aligned}
\frac{\partial \tilde{n}_{p} \delta R_{p, i j}}{\partial t}+\frac{\partial \tilde{n}_{p} \delta R_{p, i j} \tilde{u}_{p, k}}{\partial x_{k}}= & -2 \frac{\tilde{n}_{p}}{\tilde{\tau}_{p}} \delta R_{p, i j}-\tilde{n}_{p} \delta R_{p, j k} \frac{\partial \tilde{u}_{p, i}}{\partial x_{k}}-\tilde{n}_{p} \delta R_{p, i k} \frac{\partial \tilde{u}_{p, j}}{\partial x_{k}} \\
& -\frac{\partial \tilde{n}_{p} \delta Q_{p, i j k}}{\partial x_{k}} .
\end{aligned}
$$

Similarly, an equation for the RUM-HF may be written as follows

$$
\begin{aligned}
\frac{\partial \tilde{n}_{p} \delta \Theta_{p, i}}{\partial t}+\frac{\partial \tilde{n}_{p} \tilde{u}_{p, j} \delta \Theta_{p, i}}{\partial x_{j}}= & -\tilde{n}_{p}\left(\frac{1}{\tilde{\tau}_{p}}+\frac{1}{\tilde{\tau}_{\theta}}\right) \delta \Theta_{p, i}-\tilde{n}_{p} \delta \Theta_{p, j} \frac{\partial \tilde{u}_{p, i}}{\partial x_{j}} \\
& -\tilde{n}_{p} \delta R_{p, i j} \frac{\partial \widetilde{T}_{p}}{\partial x_{j}}-\frac{\partial \tilde{n}_{p} \delta \Delta_{p, i j}}{\partial x_{j}} .
\end{aligned}
$$

The first term on the r.h.s. of Eq. 18 represents the dissipation of the RUM-ST due to the drag by the fluid. The second and third terms are productions by mesoscopic velocity gradients; the last term is the transport by the third-order RUM velocity correlation. Similarly, the first term on the r.h.s. of Eq. 19 accounts for the dissipation of the RUM-HF due to heat and momentum transfers, second and third terms are productions by both mesoscopic velocity and temperature gradients and the last contribution is the transport due to the third-order RUM velocity-temperature correlation. In the framework of the ACBMM approach proposed in this study, Eqs. 18 and 19 are not resolved but rather used for the development of the secondorder moment closures. Preliminary two-dimensional Eulerian-Eulerian DNS using modeled RUM-ST in conjunction with resolved RUM-HF may be found in [10]. With the purpose of developing the algebraic closures, the equation of the evolution of the RUM kinetic energy is written [56]

$$
\frac{\partial \tilde{n}_{p} \delta \theta_{p}}{\partial t}+\frac{\partial \tilde{n}_{p} \delta \theta_{p} \tilde{u}_{p, i}}{\partial x_{i}}=-\tilde{n}_{p} \delta R_{p, i j} \frac{\partial \tilde{u}_{p, i}}{\partial x_{j}}-2 \frac{\tilde{n}_{p}}{\tilde{\tau}_{p}} \delta \theta_{p}-\frac{1}{2} \frac{\partial \tilde{n}_{p} \delta Q_{p, i}}{\partial x_{i}},
$$

where $\delta Q_{p, i}=\left\langle\delta u_{p, i}(t) \delta u_{p, j}(t) \delta u_{p, j}(t) \mid \boldsymbol{x}_{p}(t)=\boldsymbol{x} ; \mathscr{H}_{f}\right\rangle$, and an equation for the RUM temperature variance is derived

$$
\frac{\partial \tilde{n}_{p} \delta \theta_{\theta}}{\partial t}+\frac{\partial \tilde{n}_{p} \delta \theta_{\theta} \tilde{u}_{p, i}}{\partial x_{i}}=-\tilde{n}_{p} \delta \Theta_{p, i} \frac{\partial \widetilde{T}_{p}}{\partial x_{i}}-2 \frac{\tilde{n}_{p}}{\tilde{\tau}_{\theta}} \delta \theta_{\theta}-\frac{1}{2} \frac{\partial \tilde{n}_{p} \delta \Omega_{p, i}}{\partial x_{i}} .
$$

The ACBMM bases its efficiency and accuracy on the algebraic closures provided for modeling the RUM-ST and the RUM-HF; in this study, this concern is addressed.

\section{Modeling the RUM Particle Kinetic Stress Tensor (RUM-ST)}

The RUM-ST is composed of spherical and deviatoric contributions

$$
\delta R_{p, i j}=\frac{1}{3} \delta R_{p, k k} \delta_{i j}+\delta R_{p, i j}^{*}=\frac{2}{3} \delta \theta_{p} \delta_{i j}+\delta R_{p, i j}^{*}
$$

The spherical part may be predicted by means of an additional transport equation $[26,56]$ while the deviatoric contribution is given by an algebraic closure. In this work the deviatoric RUM-ST is modeled by using a polynomial representation for tensor 
functions. The polynomial representation is used in the framework of an assumption of equilibrium of the local anisotropy. Indeed, one of the most important finding of a previous study of the authors [32] was to observe that the RUM-ST is a selfsimilar tensor which means that its temporal and spatial evolutions are related to that of its trace, involving equilibrium of anisotropy. Such a behavior, revealed by using mathematical arguments, may be physically explained. In very dilute regimes, where inter-particle collisions are negligible, the dispersed phase submitted to a strong shear develops anisotropy which achieves and preserves the theoretical asymptotic maximum value as no redistribution between stresses is possible neglecting collisions. In contrast, accounting for collisions introduce a new term in the RUM stress transport equation which accounts for a redistribution effect. In the frame of the Grad's theory, this term may be modeled [53] similarly to the return-to-isotropy Rotta's model developed for the pressure-strain correlation in turbulent flows [48]. For larger values of the solid volume fraction, the dispersed phase should move away from the maximum level of anisotropy as the collision frequency increases.

Such a self-similarity assumption leads to an algebraic stress model which is an implicit and nonlinear system of equations. It is obtained defining a local RUM anisotropy tensor as

$$
b_{p, i j}^{*}=\frac{\delta R_{p, i j}}{2 \delta \theta_{p}}-\frac{1}{3} \delta_{i j}
$$

Then the equilibrium involves

$$
\frac{D}{D t} b_{p, i j}^{*}=0 \quad \text { with } \quad \frac{D}{D t}=\frac{\partial}{\partial t}+\tilde{u}_{p, k} \frac{\partial}{\partial x_{k}},
$$

which using the definition (23) gives the relation

$$
\frac{D}{D t} \delta R_{p, i j}=\frac{\delta R_{p, i j}}{\delta \theta_{p}} \frac{D}{D t} \delta \theta_{p} .
$$

Injecting Eqs. 18 and 20 into Eq. 25, and assuming equality between left hand side (1.h.s.) and r.h.s. third-order correlations, the equation takes the form

$$
\delta R_{p, i j}\left(-\frac{\delta R_{p, n m}}{2 \delta \theta_{p}} \frac{\partial \tilde{u}_{p, n}}{\partial x_{m}}\right)=-\frac{1}{2} \delta R_{p, j k} \frac{\partial \tilde{u}_{p, i}}{\partial x_{k}}-\frac{1}{2} \delta R_{p, i k} \frac{\partial \tilde{u}_{p, j}}{\partial x_{k}} .
$$

Defining the mesoscopic particle rate-of-strain and vorticity tensors as, respectively,

$$
S_{p, i j}=\frac{1}{2}\left(\frac{\partial \tilde{u}_{p, i}}{\partial x_{j}}+\frac{\partial \tilde{u}_{p, j}}{\partial x_{i}}\right), \quad \Omega_{p, i j}=\frac{1}{2}\left(\frac{\partial \tilde{u}_{p, i}}{\partial x_{j}}-\frac{\partial \tilde{u}_{p, j}}{\partial x_{i}}\right),
$$

and using the decomposition of $S_{p, i j}$ in deviatoric and spherical contributions $S_{p, i j}=$ $S_{p, i j}^{*}+\frac{1}{3} S_{p, k k} \delta_{i j}$ and the RUM anisotropy definition (23), Eq. 26 may be rearranged as

$$
\begin{aligned}
b_{p, i j}^{*}\left(-2 b_{p, n m}^{*} S_{p, n m}^{*}\right)= & -\frac{2}{3} S_{p, i j}^{*}-\left(b_{p, i k}^{*} S_{p, k j}^{*}+S_{p, i k}^{*} b_{p, k j}^{*}-\frac{2}{3} b_{p, n m}^{*} S_{p, n m}^{*} \delta_{i j}\right) \\
& +\left(b_{p, i k}^{*} \Omega_{p, k j}-\Omega_{p, i k} b_{p, k j}^{*}\right)
\end{aligned}
$$

which represents the implicit nonlinear model proposed for closing the RUM-ST. Explicit solutions are then provided using techniques well known in turbulence 
[19, 20, 64]. Hereinafter, bold notation denotes three-dimensional second-rank tensors, curly brackets \{.\} represent the tensor trace and the asterisk means traceless tensor. The matrix multiplication is then defined in a matrix notation as $\mathbf{C}=\mathbf{A B}=$ $A_{i k} B_{k j}=C_{i j}$ and $\mathbf{B}^{\mathbf{2}}=\mathbf{B B}$. According to Pope [41], the anisotropy tensor may be expressed using a polynomial representation for tensor functions $\mathbf{b}^{*}=\sum_{\varsigma} G_{\varsigma} \mathbf{T}^{(\varsigma)}$ which represents the linear combination of a set of non-dimensional independent, symmetric and deviatoric second-order tensors $\mathbf{T}^{(5)}$, using scalar coefficients $G_{\zeta}$ which are functions of the invariants of the dimensionless $\mathbf{S}^{+}$and $\mathbf{\Omega}^{+}$. Using the Cayley-Hamilton theorem, Pope [41] showed that a set of ten $(\varsigma=10)$ tensors $\mathbf{T}^{(\varsigma)}$ is needed to form an integrity basis [58] in order to express every symmetric deviatoric three-dimensional second-order tensor formed by $\mathbf{S}^{+}$and $\mathbf{\Omega}^{+}$; the problem then reduces to model the ten coefficients $G_{5}$ (see, e.g., [19, 64]). However, the integrity basis and coefficients associated with may be reduced if some approximations are introduced. Using a two-dimensional flow approximation (three tensor basis) Girimaji [20] developed a self-consistent solution technique in order to model the Reynolds stresses in turbulent flows. In this work, we will use the same technique applied to our model (28). The algebraic, implicit and nonlinear system for the dispersed phase is rewritten using the Girimaji's notation as

$$
\begin{aligned}
\mathbf{b}^{*}\left(L_{1}^{0}-L_{1}^{1}\left\{\mathbf{b}^{*} \mathbf{S}^{+}\right\}\right)= & L_{2} \mathbf{S}^{+}+L_{3}\left(\mathbf{b}^{*} \mathbf{S}^{+}+\mathbf{S}^{+} \mathbf{b}^{*}-\frac{2}{3}\left\{\mathbf{b}^{*} \mathbf{S}^{+}\right\} \mathbf{I}\right) \\
& -L_{4}\left(\mathbf{b}^{*} \boldsymbol{\Omega}^{+}-\boldsymbol{\Omega}^{+} b^{*}\right)
\end{aligned}
$$

where $L_{1}^{0}=0, L_{1}^{1}=2, L_{2}=-\frac{2}{3}, L_{3}=-1$ and $L_{4}=-1, \mathbf{S}^{+}$and $\boldsymbol{\Omega}^{+}$are dimensionless tensors by the quantity $I I_{S}^{1 / 2}=\left\{\mathbf{S}^{* \mathbf{2}}\right\}^{\mathbf{1 / 2}}$, for example, and $\mathbf{I}$ is the identity matrix. According to Girimaji [20], the general representation of the anisotropy tensor under the two-dimensional approximation is

$$
\mathbf{b}^{*}=\sum_{\varsigma=1}^{3} G_{\varsigma} \mathbf{T}^{(\varsigma)}=G_{1} \mathbf{S}^{+}+G_{2}\left(\mathbf{S}^{+} \boldsymbol{\Omega}^{+}-\boldsymbol{\Omega}^{+} \mathbf{S}^{+}\right)+G_{3}\left(\mathbf{S}^{+2}-\frac{1}{3}\left\{\mathbf{S}^{+2}\right\} \mathbf{I}\right),
$$

where the three coefficients are functions of the two invariants $\eta_{1}=\left\{\mathbf{S}^{+2}\right\}$ and $\eta_{2}=$ $\left\{\boldsymbol{\Omega}^{+2}\right\}$. Using Eq. 30 and the two-dimensional hypothesis, the contracted product $b_{p, i j}^{*} S_{p, i j}^{+}$may be written as

$$
b_{p, i j}^{*} S_{p, i j}^{+}=G_{1} S_{p, i j}^{+} S_{p, i j}^{+}=G_{1} \eta_{1} .
$$

Inserting Eqs. 30 and 31 into the system (29) yields an expression from which, comparing homogeneous 1.h.s. and r.h.s. terms, explicit solutions for the unknown coefficients may be obtained. In our particular case in which $L_{1}^{0}=0$, the coefficients $G_{2}$ and $G_{3}$ are given by

$$
G_{2}=\frac{L_{4}}{\eta_{1} L_{1}^{1}}, \quad G_{3}=-\frac{2 L_{3}}{\eta_{1} L_{1}^{1}},
$$

and $G_{1}$ reduces to the solution of a pure quadratic equation

$$
G_{1}^{2}=-\frac{1}{\eta_{1} L_{1}^{1}}\left[L_{2}+\frac{1}{3} \eta_{1} L_{3} G_{3}-2 \eta_{2} L_{4} G_{2}\right]
$$


Equation 33 accounting for the "L" coefficients leads to $G_{1}= \pm \sqrt{2 \eta_{1}+2 \eta_{2}} / 2 \eta_{1}$, which admits real solutions only for $\eta_{1}+\eta_{2} \geq 0$. Unfortunately, as $\eta_{1}$ and $\eta_{2}$ have positive and negative sign respectively, real solutions are not ensured. In order to use this model, local negative values of the discriminant are set to zero. The legitimacy of such an approximation was investigated in [30]; it was observed that negative values correspond to small magnitudes of both the particle rate-of-strain and vorticity tensors justifying the approximation $G_{1}=0$. Concerning the sign of $G_{1}$, according to Eq. 31 and the definition of the normalized production of the RUM particle kinetic energy by shear $\left(-\left\{\mathbf{b}^{*} \mathbf{S}^{*}\right\}\right.$, from Eq. 20$)$, in the simplest case it should be taken as negative (which is the most probable sign). Negative sign for $G_{1}$ is obtained in turbulence, in the domain of applicability of an equilibrium assumption of anisotropy [20]. However, for the dispersed phase interacting with turbulent flows, it is usual to have reverse energy exchanges from the RUM to the mesoscopic contribution $[33,39]$ corresponding to a reverse sign of the first-order coefficient $G_{1}$ in a two-dimensional approximation. In order to chose the right solution, a model is proposed. It stems from the observation (not shown) that the sign of the contracted

product $b_{p, i j}^{*} S_{p, i j}^{+}$, which is the same of $G_{1}$, is correlated with the sign of the third invariant of the particle rate-of-strain tensor defined as $\eta_{3}=\left\{\mathbf{S}^{+\mathbf{3}}\right\}$. According to this observation, the model becomes $G_{1}=\operatorname{sign}\left(\eta_{3}\right) \sqrt{2 \eta_{1}+2 \eta_{2}} / 2 \eta_{1}$. This model gives excellent results when a priori assessed but it should be carefully investigated by an a posteriori analysis in order to verify its validity in actual Eulerian-Eulerian simulations. Alternatively, one can assume dilute rather than very dilute regime. The latter implies that the inter-particle collision time is much larger than the particle relaxation time, so that collisions may be neglected. If the two timescales are instead of the same order of magnitude and the volume-fraction rate is small $\left(\alpha_{p}<0.01\right)$, the regime can still be considered as dilute, i.e. no modulation of the turbulence by the presence of the particles occurs, but collisions should be taken into account. In this case the model coefficient $L_{1}^{0}$ is no longer zero, as it accounts for the effects of collisions on anisotropy, and $G_{1}$ (with the right sign) is found as the solution of a third-order polynomial admitting always a real solution. This concern is not addressed by the present study; a third-order polynomial needs a criterion for the choice of the real solution which requires a further analysis. Injecting the coefficients $G_{1}, G_{2}, G_{3}$ into Eq. 30 leads to an explicit solution for the RUM anisotropy tensor and thus for the RUM-ST. A study about the realizability of the model (28) and its explicit, two dimensional, solution (30-33) is postponed to a future work. Hereinafter, this model will be referred to as 2ФEASM (by analogy with RANS turbulence models, see, e.g. [57] and references cited in).

\section{Modeling the RUM Particle Heat Flux (RUM-HF)}

In a similar way, the RUM-HF is modeled assuming similarity between the evolution of the RUM-HF and that of the square root correlation between the RUM kinetic energy $\left(\delta \theta_{p}\right)$ and the RUM temperature variance $\left(\delta \theta_{\theta}\right)$, which involves the equilibrium of the normalized heat flux. This assumption may be written as

$$
\frac{D}{D t} \frac{\delta \Theta_{p, i}}{\sqrt{\delta \theta_{p} \delta \theta_{\theta}}}=0
$$


which developed leads to

$$
\frac{1}{\sqrt{\delta \theta_{p} \delta \theta_{\theta}}} \frac{D}{D t} \delta \Theta_{p, i}=\frac{1}{2} \frac{\delta \Theta_{p, i}}{\sqrt{\delta \theta_{p} \delta \theta_{\theta}}} \frac{1}{\delta \theta_{p}} \frac{D}{D t} \delta \theta_{p}+\frac{1}{2} \frac{\delta \Theta_{p, i}}{\sqrt{\delta \theta_{p} \delta \theta_{\theta}}} \frac{1}{\delta \theta_{\theta}} \frac{D}{D t} \delta \theta_{\theta} .
$$

Then injecting Eqs. 19, 20 and 21 into Eq. 35 and assuming equality between 1.h.s. and r.h.s. diffusion terms, the equation takes the form

$$
\frac{\delta \Theta_{p, i}}{\sqrt{\delta \theta_{p} \delta \theta_{\theta}}}\left(-\frac{1}{2} \frac{\delta \Theta_{p, l}}{\delta \theta_{\theta}} \frac{\partial \widetilde{T}_{p}}{\partial x_{l}}-\frac{1}{2} \frac{\delta R_{p, k l}}{\delta \theta_{p}} \frac{\partial \tilde{u}_{p, k}}{\partial x_{l}}\right)=-\frac{\delta R_{p, i j}}{\sqrt{\delta \theta_{p} \delta \theta_{\theta}}} \frac{\partial \widetilde{T}_{p}}{\partial x_{j}}-\frac{\delta \Theta_{p, j}}{\sqrt{\delta \theta_{p} \delta \theta_{\theta}}} \frac{\partial \tilde{u}_{p, i}}{\partial x_{j}},
$$

which may be rearranged as follows

$$
\begin{aligned}
\delta H_{p, i}\left(-\frac{1}{2} \delta H_{p, l} \widetilde{K}_{p, l}-b_{p, k l}^{*} S_{p, k l}^{*}\right)= & -2\left(b_{p, i j}^{*}+\frac{1}{3} \delta_{i j}\right) \widetilde{K}_{p, j} \\
& -\left(S_{p, i j}^{*}+\Omega_{p, i j}\right) \delta H_{p, j},
\end{aligned}
$$

by defining the quantities

$$
\delta H_{p, i}=\frac{\delta \Theta_{p, i}}{\sqrt{\delta \theta_{p} \delta \theta_{\theta}}}, \quad \widetilde{K}_{p, i}=\sqrt{\frac{\delta \theta_{p}}{\delta \theta_{\theta}}} \frac{\partial \widetilde{T}_{p}}{\partial x_{i}} .
$$

A fully dimensionless system may be obtained using non-dimensionalized quantities $\widetilde{K}_{p, i}, S_{p, i j}^{*}$ and $\Omega_{p, i j}$ by the same inverse timescale, then referred to as $\widetilde{K}_{p, i}^{+}, S_{p, i j}^{+}$ and $\Omega_{p, i j}^{+}$. Eq. 37 is an implicit and nonlinear particle algebraic heat flux model. Explicit self-consistent solutions, referred to as $2 \Phi \mathrm{EAHFM,} \mathrm{are} \mathrm{then} \mathrm{provided}$ using a technique suggested for turbulent heat flux by Wikström et al. [65] and by introducing a regularization procedure for ensuring non-singular solutions during the process of matrix inversion. Equation 37 was indeed rearranged similarly to the analogous equation of Wikström et al. [65] in order to straightforwardly apply their technique to our model. Using the same notation as in [65], we define the scalar quantity into the parentheses on the 1.h.s. of Eq. 37 as

$$
N_{\theta}=-\frac{1}{2} \delta H_{p, l} \widetilde{K}_{p, l}^{+}-b_{p, k l}^{*} S_{p, k l}^{+}
$$

The system (37) may then be solved by inverting the matrix

$$
A_{p, i j}=N_{\theta} \delta_{i j}+S_{p, i j}^{+}+\Omega_{p, i j}^{+}
$$

obtaining the explicit solutions

$$
\delta H_{p, i}=-2 A_{p, i j}^{-1}\left(b_{p, j l}^{*}+\frac{1}{3} \delta_{j l}\right) \widetilde{K}_{p, l}^{+} .
$$

According to the Cayley-Hamilton theorem, an analytic expression for $\mathbf{A}^{-1}$ may be written (see, e.g., [42] ) and the operation of matrix inversion is ensured provided that A admits real non-null eigenvalues so to avoid the occurrence of singularities when its determinant (denominator of the analytical solution) vanishes. The technique of Wikström et al. [65] involves injecting the relation of the flux (Eq. 41 in our case) into the definition of $N_{\theta}$ (Eq. 39 in our case), replacing $\mathbf{A}^{-1}$ by its analytical 
expression. For fully three-dimensional flows, this leads to a fourth-order polynomial in $N_{\theta}$ difficult to handle. An alternative is to resolve a third-order polynomial corresponding to a two-dimensional flow approximation and then using the solution for three-dimensional flows as well [65]. Assuming two-dimensional flows, the analytic expression for the inverse of the matrix is

$$
\mathbf{A}^{-1}=\frac{N_{\theta} \mathbf{I}-\left(\mathbf{S}^{+}+\boldsymbol{\Omega}^{+}\right)}{N_{\theta}^{2}-\frac{1}{2}\left(\eta_{1}+\eta_{2}\right)} .
$$

Injecting Eq. 42 (but using three-dimensional tensors) into Eq. 41 and the latter into the definition (39) the following third-order polynomial is obtained

$$
2 N_{\theta}^{3}+2\left\{\mathbf{b}^{*} \mathbf{S}^{+}\right\} N_{\theta}^{2}-\left(Q_{1}+R_{1}\right) N_{\theta}-\left\{\mathbf{b}^{*} \mathbf{S}^{+}\right\} Q_{1}+R_{2}=0,
$$

where $Q_{1}=\eta_{1}+\eta_{2}$ and the terms $R_{1}$ and $R_{2}$ are, respectively,

$$
\begin{aligned}
& R_{1}=2\left(\left(b_{p, i l}^{*}+\frac{1}{3} \delta_{i l}\right) \widetilde{K}_{p, l}^{+} \widetilde{K}_{p, i}^{+}\right) \\
& R_{2}=2\left(\left(S_{p, i j}^{+}+\Omega_{p, i j}^{+}\right)\left(b_{p, j l}^{*}+\frac{1}{3} \delta_{j l}\right) \widetilde{K}_{p, l}^{+} \widetilde{K}_{p, i}^{+}\right) .
\end{aligned}
$$

A unique real solution is ensured by using the transcendental functions

$$
\begin{aligned}
N_{\theta}= & \frac{-\left\{\mathbf{b}^{*} \mathbf{S}^{+}\right\}}{3} \\
& + \begin{cases}\operatorname{sign}\left(P_{1}+\sqrt{P_{2}}\right)\left|P_{1}+\sqrt{P_{2}}\right|^{1 / 3}+\operatorname{sign}\left(P_{1}-\sqrt{P_{2}}\right)\left|P_{1}-\sqrt{P_{2}}\right|^{1 / 3}, & P_{2} \geq 0 \\
2\left(P_{1}^{2}-P_{2}\right)^{1 / 6} \cos \left(\frac{1}{3} \arccos \left(\frac{P_{1}}{\sqrt{P_{1}^{2}-P_{2}}}\right)+\beta\right), & P_{2}<0, P_{1} \geq 0 \\
2\left(P_{1}^{2}-P_{2}\right)^{1 / 6} \cos \left(-\frac{1}{3} \arccos \left(\frac{-P_{1}}{\sqrt{P_{1}^{2}-P_{2}}}\right)+\frac{\pi}{3}+\beta\right), & P_{2}<0, P_{1}<0\end{cases}
\end{aligned}
$$

with $P_{1}$ and $P_{2}$ defined by the relations

$$
\begin{aligned}
& P_{1}=\left(\frac{-\left\{\mathbf{b}^{*} \mathbf{S}^{+}\right\}^{3}}{27}+\frac{1}{6}\left\{\mathbf{b}^{*} \mathbf{S}^{+}\right\} Q_{1}-\frac{1}{12}\left\{\mathbf{b}^{*} \mathbf{S}^{+}\right\} R_{1}-\frac{1}{4} R_{2}\right) \\
& P_{2}=P_{1}^{2}-\left(\frac{\left\{\mathbf{b}^{*} \mathbf{S}^{+}\right\}^{2}}{9}+\frac{1}{6} Q_{1}+\frac{1}{6} R_{1}\right)^{3}
\end{aligned}
$$

and $\beta=0$ for $-\left\{\mathbf{b}^{*} \mathbf{S}^{+}\right\} \geq 0$ and $\beta=2 \pi / 3$ for $-\left\{\mathbf{b}^{*} \mathbf{S}^{+}\right\}<0$. Transcendental functions are chosen in order to ensure the physical real solution when the temperature gradient vanish and the model must give $N_{\theta}=-\left\{\mathbf{b}^{*} \mathbf{S}^{+}\right\}$even in the case of negative production $\left(-\left\{\mathbf{b}^{*} \mathbf{S}^{+}\right\}<0\right)$, case which rarely occurs in turbulence. The solution for $N_{\theta}$ is then inserted into the Eq. 42 and $\mathbf{A}^{-1}$ injected into the system (41) which provides explicit and self-consistent solutions for the RUM-HF. At this point, we need to address the concern of stable solutions by ensuring the determinant of the matrix does not vanish and singularities do not occur. From the a priori analysis, we observed that the determinant is mainly positive and that negative values correspond to small magnitudes. In order to avoid singular solutions, the determinant should not change its sign and the following condition, $\operatorname{det}(\mathbf{A})=N_{\theta}^{2}-\frac{1}{2}\left(\eta_{1}+\eta_{2}\right)>0$, should be 
satisfied while, in general, it is not. Solutions proposed in [65] for turbulent flows do not apply to the dispersed phase and a regularization procedure is therefore necessary. The reciprocal of the determinant is re-written as follows

$$
\operatorname{det}(\mathbf{A})^{-1}=\frac{1}{N_{\theta}^{2}-\frac{1}{2}\left(\eta_{1}+\eta_{2}\right)}=\frac{2 /\left(2 N_{\theta}^{2}\right)}{1-\eta^{2} /\left(2 N_{\theta}^{2}\right)+\xi^{2} /\left(2 N_{\theta}^{2}\right)}=\frac{2 /\left(2 N_{\theta}^{2}\right)}{1+\xi^{2} /\left(2 N_{\theta}^{2}\right)-x^{2}}
$$

where $\eta=\sqrt{\eta_{1}}, \xi=\sqrt{-\eta_{2}}$ and $x^{2}=\eta^{2} /\left(2 N_{\theta}^{2}\right)$. Then, using the first order Maclaurin series $^{2}$ of the function $1 /\left(1+x^{2}\right)$ we write $x^{2} \approx 1-1 /\left(1+x^{2}\right)$ which inserted into Eq. 47 leads to

$$
\operatorname{det}(\mathbf{A})^{-1}=\frac{2\left(2 N_{\theta}^{2}+\eta^{2}\right)}{\left(2 N_{\theta}^{2}\right)^{2}+\xi^{2}\left(2 N_{\theta}^{2}+\eta^{2}\right)} .
$$

The stability is thus ensured as the denominator of Eq. 48 never vanishes for $N_{\theta}$ and $\xi^{2}$ or $\eta^{2} \neq 0$. An analysis conducted by comparing scatter plots of both the non regularized solutions and the regularized solutions versus the actual values has shown that the regularization procedure has an impact mainly on the predictions of the small magnitude of the RUM-HF and slightly affects the model accuracy. Hereinafter, explicit regularized solutions for the RUM-HF will be referred to as $2 \Phi E A H F M$.

\section{Results}

An evaluation of the ACBMM by assessing the proposed algebraic closures is given by an a priori analysis using particle Eulerian fields which are extracted from several Lagrangian-Eulerian DNSs of a temporal particle-laden non-isothermal turbulent planar jet. The particle Eulerian database is obtained from the Lagrangian-Eulerian DNSs using the projection procedure as detailed in [26, 39]. Mesoscopic Eulerian fields are then used for assessed the models by means of point-wise functions (as, for example, PDF) over the local particle Eulerian quantities, or by using mean profiles of the Eulerian fields, computed by density-weighted averaging the Eulerian fields over the homogeneity planes of the planar jet.

The numerical simulations correspond to the dispersion of a cold particle-laden jet into a hot homogeneous isotropic decaying turbulence [2]. The gas is solved by compressible Navier-Stokes and energy equations in dimensionless form using a third order Runge-Kutta time stepping and a sixth-order compact finite difference scheme on a Cartesian mesh. The simulation domain is a cube of length size $L_{\text {box }}=2 \pi L_{\text {ref }}$ and grid $128^{3}$ cells. The initial gas velocity has hyperbolic-tangent profile supplemented with statistically homogeneous and isotropic velocity fluctuations [61]. The initial gas temperature has hyperbolic-tangent mean profile and zero fluctuations. As we assume very dilute regime, collisions and turbulence modulation are not accounted for. The advancement in time of the Lagrangian tracking is ensured by a third order Runge-Kutta scheme. The interpolation of the instantaneous local fluid

\footnotetext{
${ }^{2}$ A similar regularization procedure was used by Gatski and Speziale [19] for modeling the Reynolds stress tensor in the framework of the EASM approach.
} 
velocity and temperature at the particle location is provided at any time stepping by a third-order Lagrange polynomial algorithm. Periodic boundary conditions are applied to both the carrier and the dispersed phases. Within the slab, of width $d=0.25 L_{\mathrm{box}}, 13$ millions of solid particles are randomly embedded at the same mean velocity and temperature as the carrier flow and zero fluctuations. Seven simulations are conducted corresponding to the macroscale-turbulent Stokes number St $\sim 0.1,0.5,1,2,3,5,7$ computed over a characteristic timescale of the turbulence seen by the particles, $\tau_{f @ p}^{L}$. The timescale $\tau_{f @ p}^{L}$ is obtained assuming the Tchen equilibrium in the spanwise direction of the jet (mean-flow free) [43] for a simulation of reference $(\mathrm{St} \sim 1)$. An estimate of the Stokes numbers is then obtained as the ratio between the mean particle relaxation times, computed changing uniquely the particle density, and $\tau_{f @ p}^{L}$. As the particles are very small and heavy, the drag correction has only a little impact on the computation of the particle relaxation time and the regime is very close to the Stokes regime. As a consequence, according to Eq. 4 and the parameters chosen for the numerical simulations, $\operatorname{Pr}=0.7$ and $\alpha=2$, the thermal particle relaxation times result in about twice the dynamic particle relaxation times. Assuming equality between the characteristic dynamic and thermal timescales of the turbulence seen by the particles, then $\mathrm{St}_{\theta} \sim 2 \mathrm{St}$. This implies that the thermal particle inertia is even larger than the dynamic particle inertia. The initial set of dimensionless parameters of the numerical simulations is the following: kinematics viscosity $v_{f}=1.82 \mathrm{e}-4 u_{\text {ref }} L_{\text {ref }}$, turbulent kinetic energy $q_{f}^{2}=3.37 \mathrm{e}-4 u_{\text {ref }}^{2}$, dissipation $\epsilon_{f}=3.78 \mathrm{e}-5 u_{\text {ref }}^{3} / L_{\text {ref }}$, jet mean velocity $U_{f}=0.15 u_{\text {ref }}$, jet rms velocity $u_{f}^{\prime}=0.015 u_{\text {ref }}$, jet temperature $T_{f}=0.8 T_{\text {ref }}$, particles' diameter $d_{p} / \Delta_{x}=0.01$. For all the numerical simulations, at the final time, the Reynolds number based on the large lengthscale of the turbulence, $\operatorname{Re}_{L}=\left(q_{f}^{2}\right)^{2} /(\epsilon \nu)$, is $\operatorname{Re}_{L} \sim 80$ at the center of the jet and almost the double in the zones of high shear. Hereinafter, quantities are shown dimensionless.

The evaluation of the accuracy of the models is performed at tensor/vector level by assessing each component of the deviatoric RUM-ST and the RUM-HF against the actual quantities as provided by the particle Eulerian database (not all shown for brevity). Moreover, an assessment at scalar level is also given. Illustrated in Fig. 1 are the mean profiles of $\delta R_{p, 11}^{*}$, as obtained using 2ФEASM, and that of the corresponding actual stresses, for all the Stokes-number simulations. Comparisons
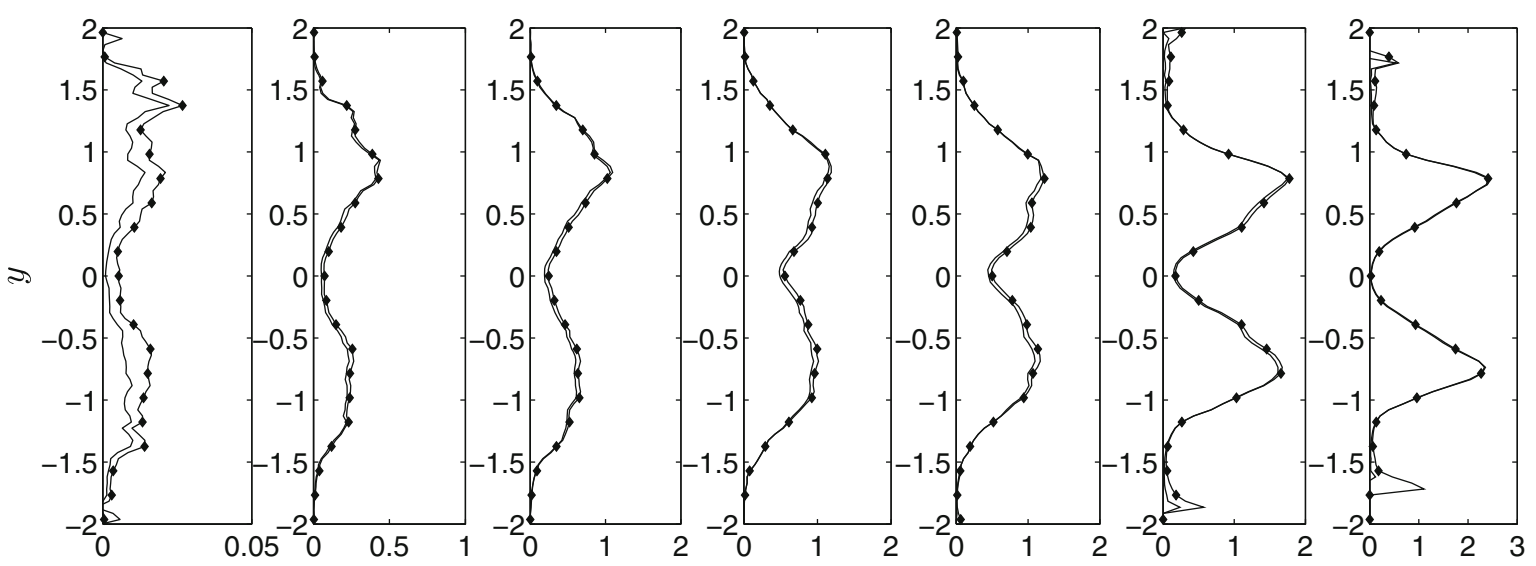

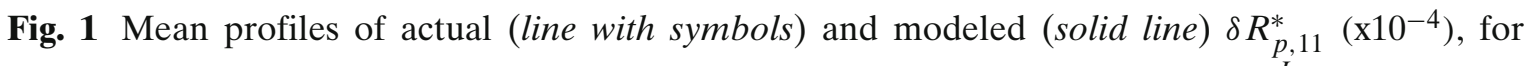
simulations corresponding, from left to right, to St $\sim 0.1,0.5,1,2,3,5,7$, at $t=6.2 \tau_{f @ p}^{L}$ 

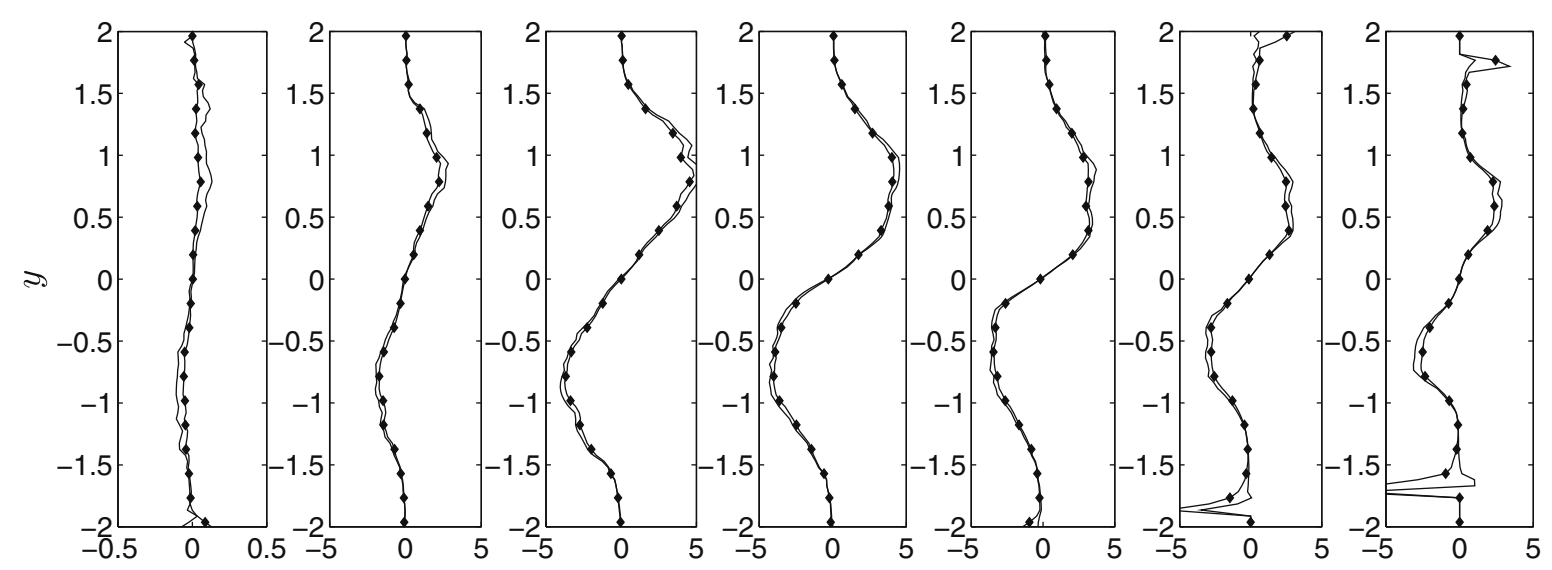

Fig. 2 Mean profiles of actual (line with symbols) and modeled (solid line) $\delta R_{p, 12}^{*}\left(\mathrm{x} 10^{-5}\right.$ ), for simulations corresponding, from left to right, to $\mathrm{St} \sim 0.1,0.5,1,2,3,5,7$, at $t=6.2 \tau_{f @ p}^{L}$

show that, except for the smallest Stokes-number simulation where the model slightly underestimate the mean profile of $\delta R_{p, 11}^{*}$, mean quantities are excellently reproduced regardless of the particle inertia. Illustrated in Fig. 2 are the modeled and the actual mean profiles of the component $\delta R_{p, 12}^{*}$. Contrary to $\delta R_{p, 11}^{*}$, the mean profile of the component $\delta R_{p, 12}^{*}$ is slightly overestimated for the smallest Stokes-number simulation and like $\delta R_{p, 11}^{*}$, mean profiles of $\delta R_{p, 12}^{*}$ are excellently reproduced by the model independent of the simulation. In Fig. 3, 2ФEASM is assessed at the scalar level over the deviatoric-RUM contribution of the first r.h.s. term of Eq. 20 which represents the production of the RUM kinetic energy by shear $\left(P_{\mathrm{RUM}-\mathrm{KE}}\right)$. Results show that the mean amount of $P_{\mathrm{RUM}-\mathrm{KE}}$ is globally overestimated by the model. In order to gain insight into the local prediction of the deviatoric RUM-ST and the $P_{\mathrm{RUM}-\mathrm{KE}}$, the joint PDFs of the actual and modeled $\delta R_{p, 11}^{*}$ and the actual and modeled $P_{\mathrm{RUM}-\mathrm{KE}}$ are computed and the results (for an intermediate Stokesnumber simulation, St $\sim 3$ ) depicted in Fig. 4. Examination of the Fig. 4a shows that $2 \Phi E A S M$ reproduces very well the component $\delta R_{p, 11}^{*}$ of the tensor, being the values equilikely distributed around the 45 degree line which represents the perfect agreement between the two quantities. Examination of the Fig. $4 \mathrm{~b}$ shows that also

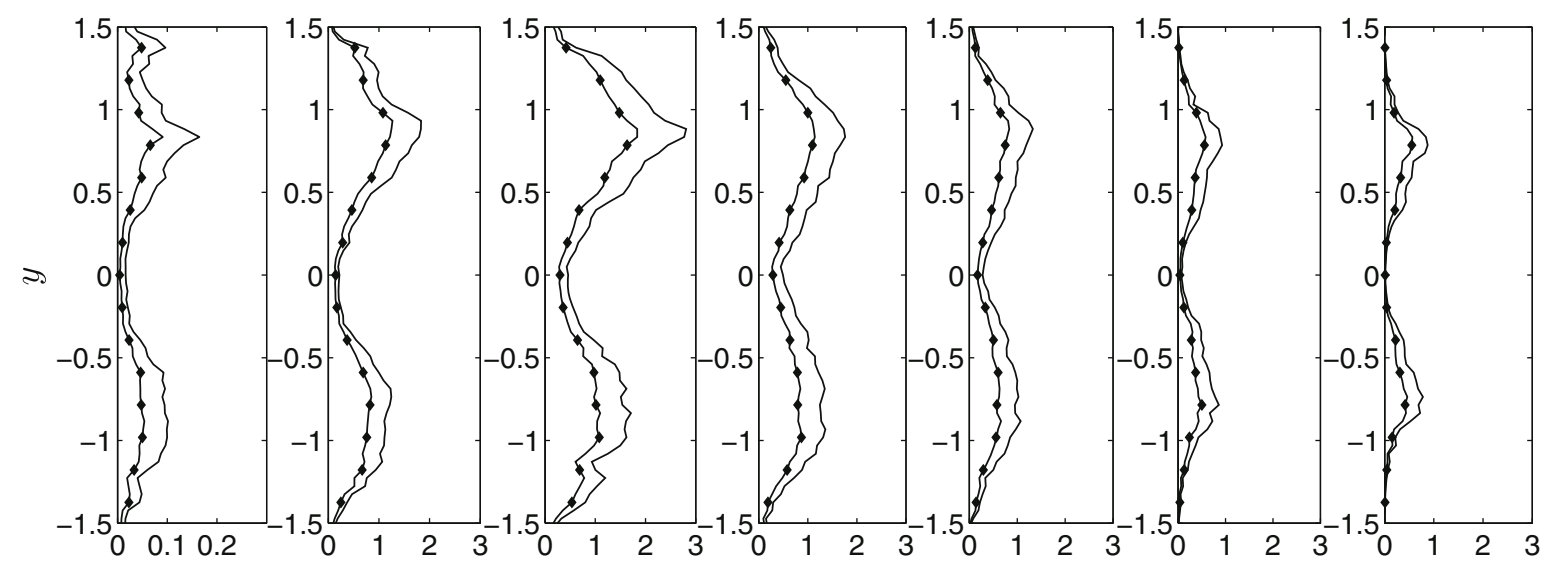

Fig. 3 Mean profiles of actual (line with symbols) and modeled (solid line) $P_{\mathrm{RUM}-\mathrm{KE}}\left(\mathrm{x} 10^{-5}\right.$ ), for simulations corresponding, from left to right, to $\mathrm{St} \sim 0.1,0.5,1,2,3,5,7$, at $t=6.2 \tau_{\text {f@ }}^{L} p$ 

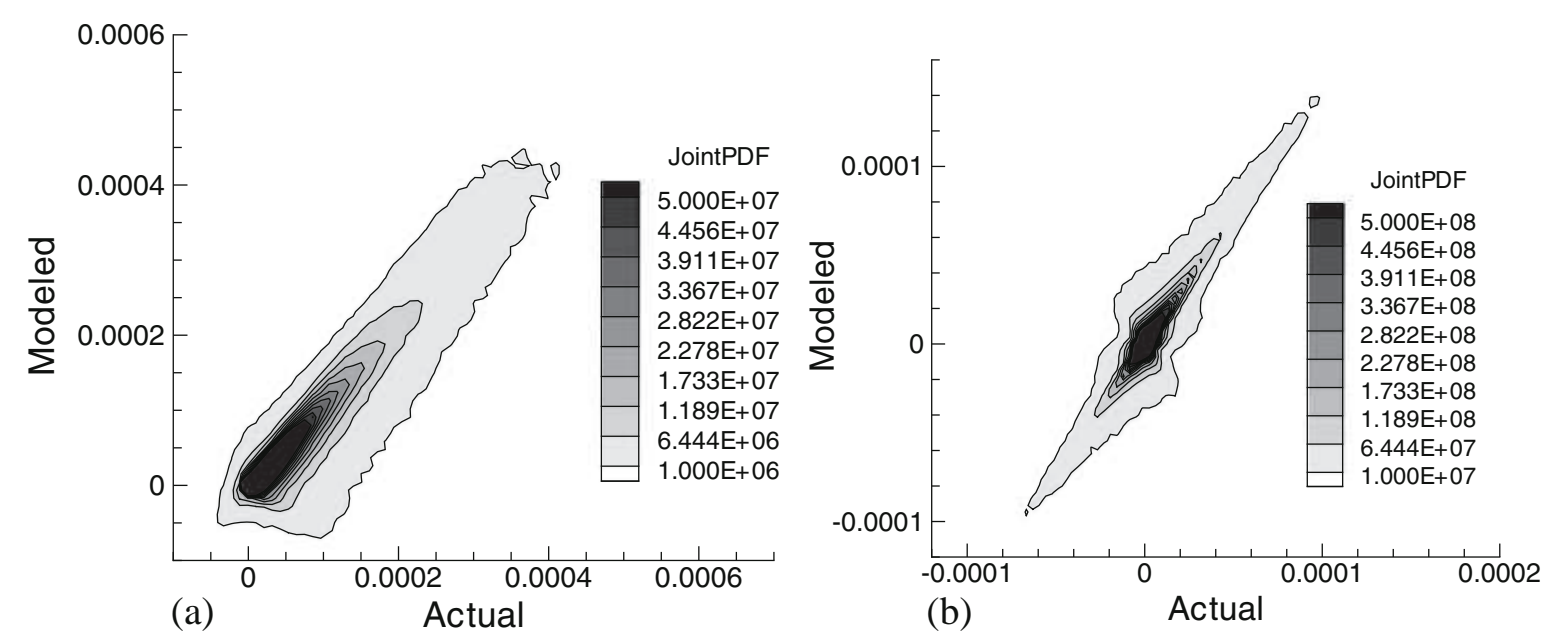

Fig. 4 Isocontours of the joint PDF of (a) modeled and actual $\delta R_{p, 11}^{*}$, (b) modeled and actual $P_{\mathrm{RUM}-\mathrm{KE}}$, computed into the slab, $-0.7<y^{*}<0.7$, for the simulation corresponding to $\mathrm{St} \sim 3$, at the time $t=6.2 \tau_{f @ p}^{L}$

the joint-PDF values of the actual and modeled productions are mainly equilikely distributed around a line, the slope of which is however larger than 45 degree, which means that the magnitudes of the modeled $P_{\mathrm{RUM}-\mathrm{KE}}$ are overestimated. Since positive values dominate negative values, the averages over planes have positive sign (see Fig. 3) and an overestimate of the magnitudes corresponds to an overestimate of the mean profiles as well. In order to give a more quantitative evidence of such a behavior, the PDF of $P_{\mathrm{RUM}-\mathrm{KE}}$ is investigated and the result corresponding to the simulation St 3 is displayed in Fig. 5. Examination of the Fig. 5 shows that the predicted $P_{\mathrm{RUM}-\mathrm{KE}}$ is larger in magnitude than the reference; nevertheless, the shape of the PDF is perfectly reproduced. As a perspective for future work, the model could be improved using a three-dimensional form (ten tensor basis), but then the question of non-singular solutions should be addressed. Also, the model may be improved assuming dilute rather then very dilute regime (as pointed out in Section 3).

As the aim of this work is to provide a comprehensive model for predicting non-isothermal particle-laden turbulent flows in a Eulerian fashion, the assessment

Fig. 5 PDF of actual (line with symbols) and modeled (solid line) $P_{\mathrm{RUM}-\mathrm{KE}}$, computed into the slab, $-0.7<y^{*}<0.7$, for the simulation corresponding to St $\sim 3$, at $t=6.2 \tau_{f @ p}^{L}$, evaluated in the range of \pm 1 the standard deviation from the mean value

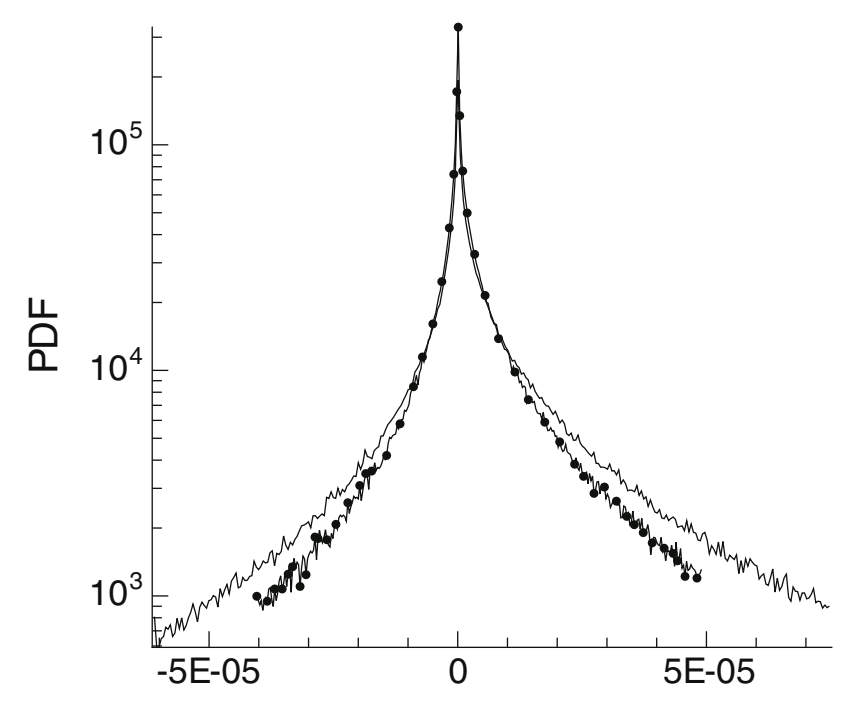




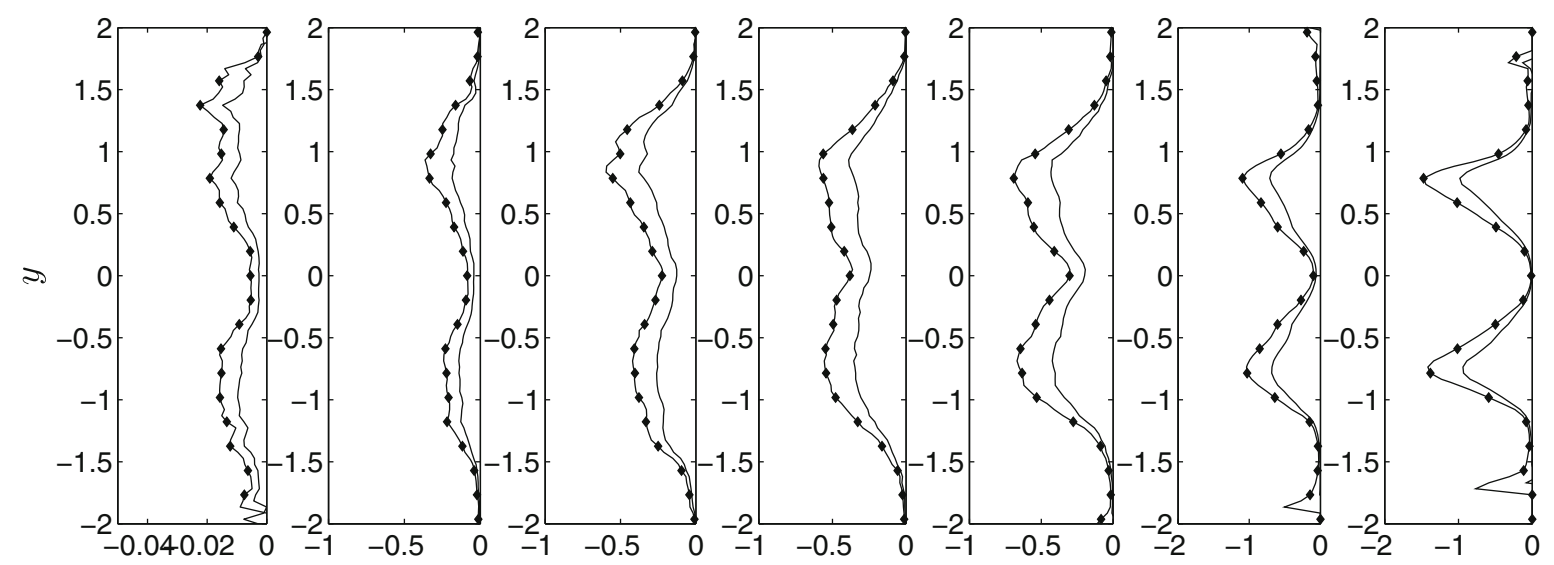

Fig. 6 Mean profiles of actual (line with symbols) and modeled (solid line) $\delta \Theta_{p, 1}\left(\mathrm{x} 10^{-3}\right)$, for simulations corresponding, from left to right, to $\mathrm{St} \sim 0.1,0.5,1,2,3,5,7$, at $t=6.2 \tau_{f @ p}^{L}$

of the RUM-HF closure is performed by using, as an input, the RUM-ST as modeled by $2 \Phi E A S M$. Results of the predictions using $2 \Phi E A H F M$ for the streamwise and the normal components of the RUM-HF are depicted in Figs. 6 and 7. Moreover, in order to give an assessment of the model at the scalar level, the scalar quantity corresponding to the first term in the r.h.s. of Eq. 21, namely the production of the RUM temperature variance ( $\left.P_{\mathrm{RUM}-\mathrm{TV}}\right)$ is investigated. Meanprofile results show that the model tends to underestimate the flux components while it better reproduces the mean production amount (Fig. 8). A local (notaveraged) representation of the model predictions for the $P_{\text {RUM-TV }}$ is given by the PDF and the joint PDF in Figs. 9 and 10a, respectively. Examination of the Fig. 9 shows that the slight overestimation of the modeled $P_{\mathrm{RUM}-\mathrm{TV}}$, as observed by the mean profiles, is likely due to an underestimation of the negative contribution of $P_{\text {RUM-TV }}$ corresponding to small values of it; nevertheless the shape of the PDF is very well reproduced. Good agreement between modeled and actual productions is also observed by the examination of the Fig. 10a. In order to gain insight into the point-wise predictions of the RUM-HF components, the joint PDF of the actual and modeled $\delta \Theta_{p, 1}$ is computed and displayed in Fig. 10b. Examination of the
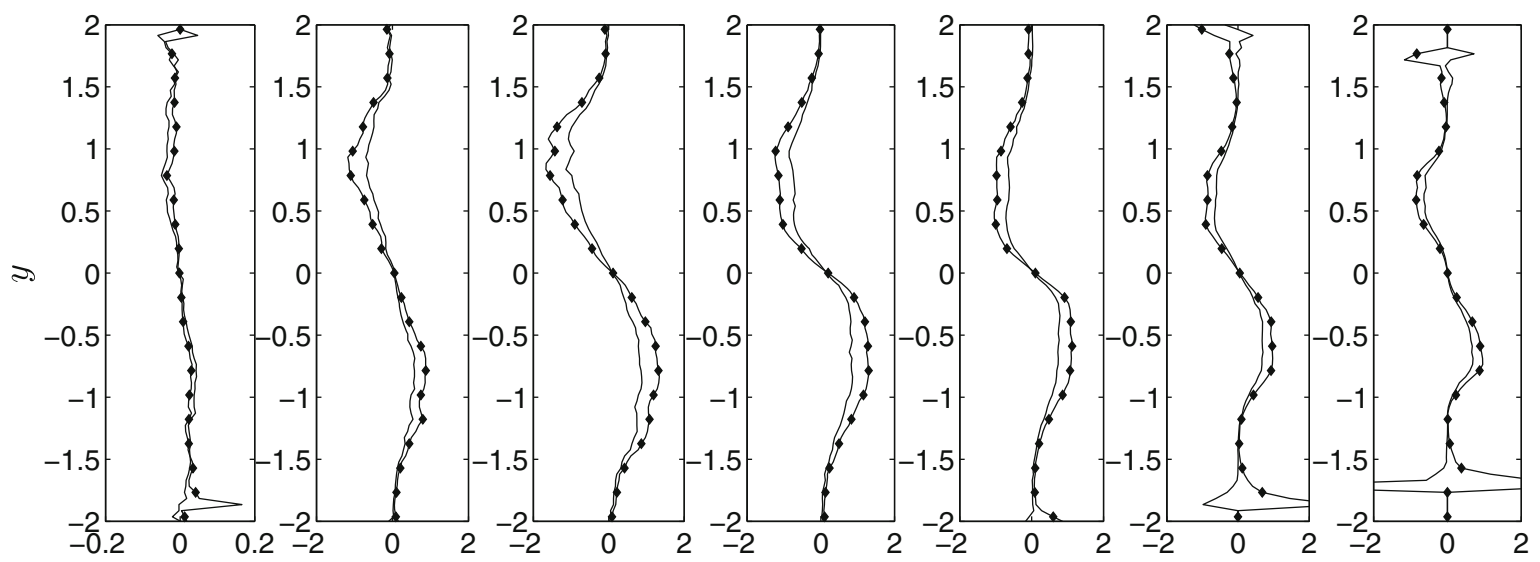

Fig. 7 Mean profiles of actual (line with symbols) and modeled (solid line) $\delta \Theta_{p, 2}\left(\mathrm{x} 10^{-4}\right)$, for simulations corresponding, from left to right, to St $\sim 0.1,0.5,1,2,3,5,7$, at $t=6.2 \tau_{f @ p}^{L}$ 


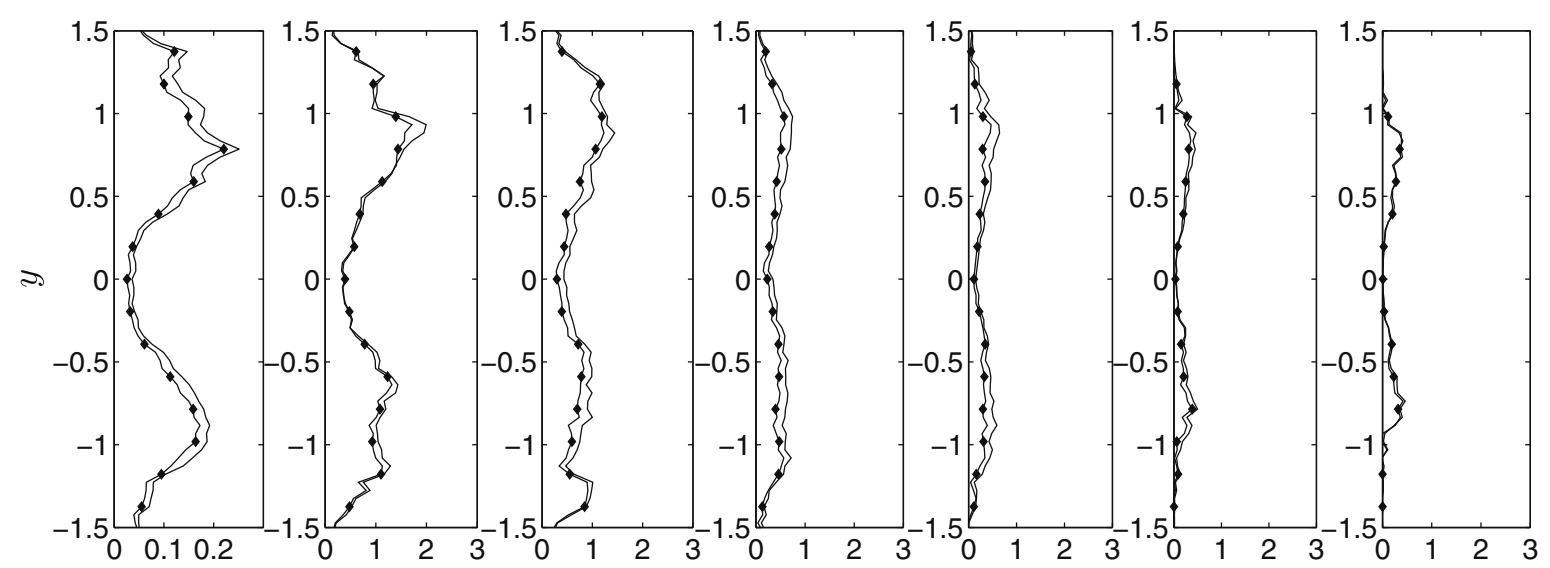

Fig. 8 Mean profiles of actual (line with symbols) and modeled (solid line) $P_{\mathrm{RUM}-\mathrm{TV}}\left(\mathrm{x} 10^{-4}\right.$ ), for simulations corresponding, from left to right, to St $\sim 0.1,0.5,1,2,3,5,7$, at $t=6.2 \tau_{f @ p}^{L}$

Fig. 9 PDF of actual (line with symbols) and modeled (solid line) $P_{\text {RUM-TV }}$ computed into the slab, $-0.7<y^{*}<0.7$, for the simulation corresponding to $\mathrm{St}$ $\sim 3$, at $t=6.2 \tau_{f @ p}^{L}$, evaluated in the range of \pm 1 the standard deviation from the mean value

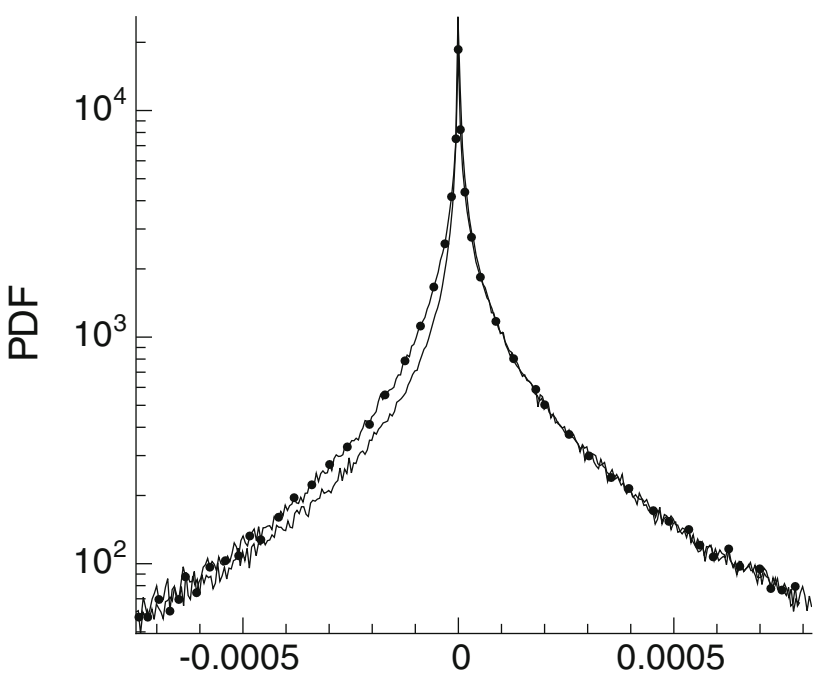

results shows that this component is very well predicted at intermediate and large
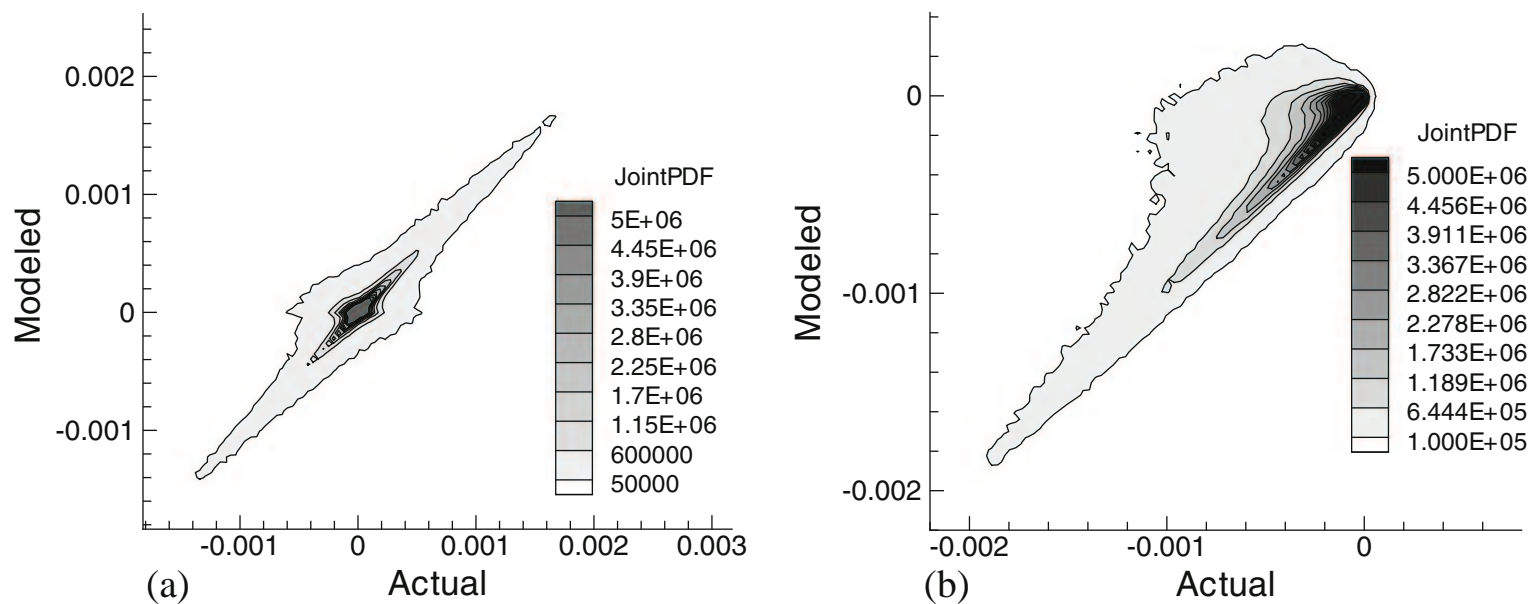

Fig. 10 Isocontours of the joint PDF of (a) modeled and actual $P_{\text {RUM-TV }},($ b) modeled and actual $\delta \Theta_{p, 1}$, computed into the slab, $-0.7<y^{*}<0.7$, for the simulation corresponding to $\mathrm{St} \sim 3$, at the time $t=6.2 \tau_{f @ p}^{L}$ 


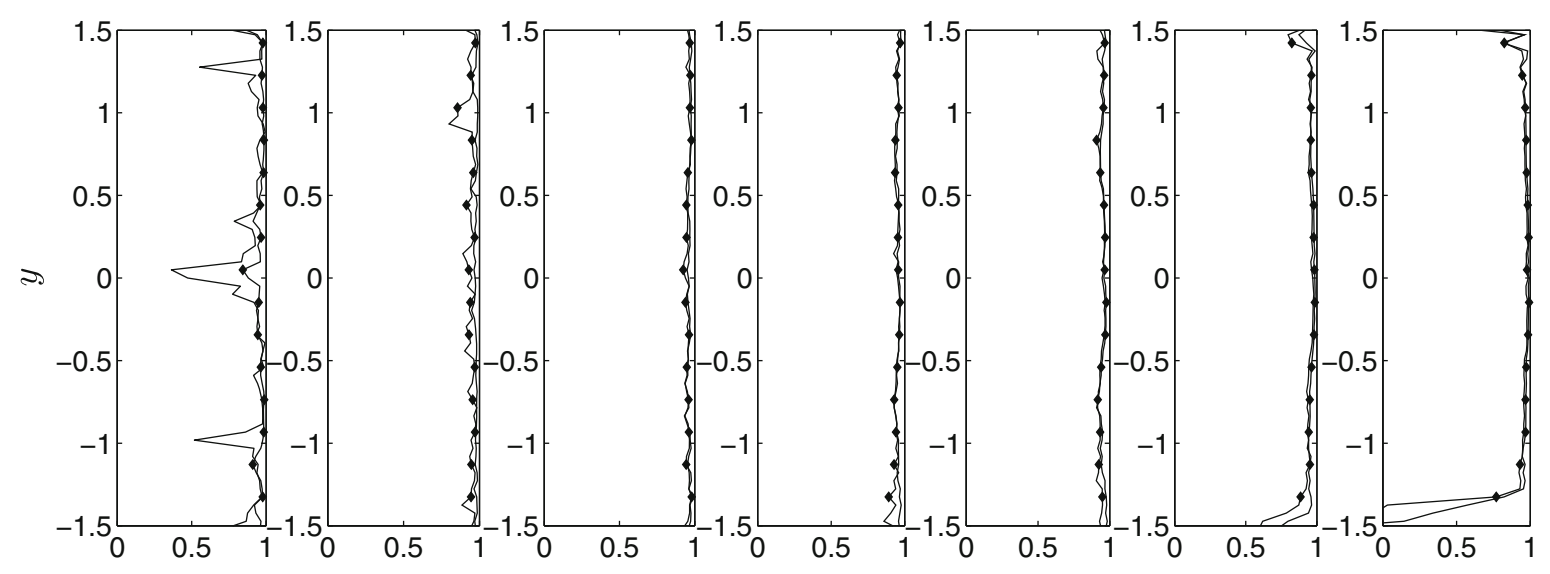

Fig. 11 Correlation coefficients computed over modeled and actual quantities $P_{\mathrm{RUM}-\mathrm{KE}}$ (solid line) and $P_{\mathrm{RUM}-\mathrm{TV}}$ (line with symbols) for simulations corresponding, from left to right, to St $\sim 0.1,0.5,1,2,3,5,7$, at $t=6.2 \tau_{f @ p}^{L}$

magnitudes while worse predictions are found close to the small values of the RUMHF. It is conjectured that small values correspond to the zones of weak temperature/velocity gradients where a two-dimensional approximation is more questionable. The model could be improved by using a full three-dimensional writing together with a new regularization procedure for the matrix inversion. Globally the two closures give satisfactory results. Correlation coefficients between modeled and actual $P_{\mathrm{RUM}-\mathrm{KE}}$ and $P_{\mathrm{RUM}-\mathrm{TV}}$ respectively, computed according to the relation $C(A, B)=$ $(<A B>-<A><B>) /\left(\sqrt{\left(<A^{2}>-<A>^{2}\right)\left(<B^{2}>-<B>^{2}\right)}\right)$, are depicted in Fig. 11; they show the high-fidelity representation by the models of the actual quantities.

\section{Conclusions}

An algebraic-closure-based moment method has been developed for unsteady nonisothermal Eulerian particle simulations coupled with DNS of carrier fluid turbulent flows in very dilute regime and at moderate turbulent-macroscale Stokes numbers. This method relies on a conditional statistical approach which provides a local characterization of the dispersed phase as it accounts for the effect of the crossing between particle trajectories which occurs in the presence of inertial particles. The ACBMM approach proposed by this work entails the numerical integration of a set of closed conservation equations describing the evolution of the low-order moments of the conditional PDF, namely the mesoscopic particle number density, velocity and temperature. Closures for the second-order moments, the RUM-ST and the RUM$\mathrm{HF}$, appearing in the particle momentum and enthalpy equations are then provided by means of additional transport equations for the trace of the RUM stress tensor and for the RUM temperature variance together with algebraic closures derived for modeling the deviatoric part of the RUM tensor and the RUM heat flux. The deviatoric RUM-ST is closed by a polynomial representation for tensor functions used in the frame of an assumption of equilibrium of the RUM anisotropy. The RUMHF is modeled assuming equilibrium of the scaled heat flux. Explicit self-consistent solutions of the novel implicit models are then provided by using techniques well- 
known in turbulence. An a priori analysis conducted by this study has shown that these models compare very well with the actual particle Eulerian quantities extracted from Lagrangian-Eulerian DNSs, and this for all the Stokes-number simulations. At the tensor/vector level, they provide an excellent representation of the RUM-ST and a satisfactory representation of the RUM-HF, almost independent of the particle inertia. At the scalar level, the analysis based on the local scalar quantities (the RUM kinetic energy and the RUM temperature variance productions) also showed satisfactory results. However, a detailed analysis conducted on the solely fluctuating velocity/temperature contribution of these two terms (not shown) has pointed out as the accuracy of the predictions of the RUM productions by gradients of fluctuating velocity/temperature, degenerates at large Stokes numbers in high shear zones of the jet. These terms, responsible for the energy exchange between mescoscopic and RUM contributions, at large inertia and in high shear zones of the jet, tend toward zero-mean values despite their high local magnitudes (this concern makes the object of a following publication). A kinetic-energy transfer rate which is badly predicted may dramatically affect the numerical simulations. This point seems to represent the limit of a first-order modeling using second-order constitutive relations. For this reason, the model we propose is expected to be effective for dilute particleladen turbulent flows at moderate turbulent-macroscale Stokes numbers $(S t<10)$. For dilute particle-laden flows at large/very large Stokes numbers, a ACBMM approach involving higher-order-moment conservation equations or QBMM/KBMM approaches should be preferred. The a priori analysis conducted by the present study should be completed by an a posteriori assessment through actual Eulerian-Eulerian DNSs in order to give a comprehensive evaluation of the modeling and its range of applicability.

Acknowledgements This work received funding from the European Community through the TIMECOP-AE project (Project \# AST5-CT-2006-030828). It reflects only the author's views and the Community is not liable for any use that may be made of the information contained therein. Numerical simulations were performed by the IBM Power6 machine; support from Institut de Développment et des Ressources en Informatique Scientifique (IDRIS) is gratefully acknowledged.

\section{References}

1. Balzer, G., Simonin, O., Boelle, A., Laviéville, J.: A unifying modelling approach for the numerical prediction of dilute and dense gas-solid two-phase flows. In: Kwauk, M., Li, J. (eds.) Proc. of the 5th Int. Conf. on Circulating Fluidized Beds Circulating Fluidized Bed Technology V, pp. 432-439. Science Press, Beijing (1996)

2. Bédat, B., Simonin O.: Numerical study of the inter-phase heat transfers in a non-isothermal particle laden slab flow. In: Proceedings of the 5th International Conference on Multiphase Flow (ICMF). Yokohama, Japan, 30 May-4 June 2004

3. Chalons, C., Fox, R.O., Massot, M.: A multi-Gaussian quadrature method of moments for gasparticle flows in a les framework. In: Proceedings of the Center for Turbulence Research (CTR) Summer Program, pp. 347-358 (2011)

4. Chalons, C., Kah, D., Massot, M.: Beyond pressureless gas dynamics : Quadrature-based velocity moment models. Commun. Math. Sci. 10, 1241-1272 (2012)

5. Chapman, S., Cowling, T.G.: The Mathematical Theory of Non-Uniform Gases, 3rd edn. Cambridge University Press (1970)

6. Chen, C.P., Wood, P.E.: A turbulence closure model for dilute gas-particle flows. Can. J. Chem. Eng. 63(3), 349-360 (1985) 
7. Chen, H., Orszag, S.A., I.Staroselsky, Succi, S.: Expanded analogy between Boltzmann kinetic theory of fluids and turbulence. J. Fluid Mech. 519, 301-314 (2004)

8. de Chaisemartin, S., Fréret, L., Kah, D., Laurent, F., Fox, R.O., Reveillon, J., Massot, M.: Eulerian models for turbulent spray combustion with polydispersity and droplet crossing. C. R. Mecanique 337, 438-448 (2009). Special Issue Combustion for Aerospace Propulsion

9. Desjardins, O., Fox, R.O., Villedieu, P.: A quadrature-based moment method for dilute fluidparticle flows. J. Comput. Phys. 227(4), 2514-2539 (2008)

10. Dombard, J., Leveugle, B., Selle, L., Reveillon, J., Poinsot, T., D'Angelo, Y.: Modeling heat transfer in dilute two-phase flows using the mesoscopic Eulerian formalism. Int. J. Heat Mass Transf. 55(5-6), 1486-1495 (2012)

11. Druzhinin, O.A., Elghobashi, S.: Direct numerical simulations bubble-laden turbulent flows using the two-fluid formulation. Phys. Fluids 10(3), 685-697 (1998)

12. Druzhinin, O.A., Elghobashi, S.: On the decay rate of isotropic turbulence laden with microparticles. Phys. Fluids 11(3), 602-610 (1999)

13. Elghobashi, S.E., Abou-Arab, T.W.: A two-equation turbulence model for two-phase flows. Phys. Fluids 26(4), 931-938 (1983)

14. Ferry, J., Balachandar, S.: A fast Eulerian method for dispersed two-phase flow. Int. J. Multiphase Flow 27, 1199-1226 (2001)

15. Ferry, J., Balachandar, S.: Equilibrium Eulerian approach for predicting the thermal field of a dispersion of small particles. Int. J. Heat Mass Transf. 48, 681-689 (2005)

16. Février, P., Simonin, O., Squires, K.D.: Partitioning of particle velocities in gas-solid turbulent flows into a continuous field and a spatially uncorrelated random distribution: theoretical formalism and numerical study. J. Fluid Mech. 533 1-46 (2005)

17. Fox, R.O.: A quadrature-based third-order moment method for dilute gas-particle flows. J. Comput. Phys. 227(12), 6313-6350 (2008)

18. Fox, R.O., Laurent, F., Massot, M.: Numerical simulation of spray coalescence in an Eulerian framework: direct quadrature method of moments and multi-fluid method. J. Comput. Phys. 227(6), 3058-3088 (2008)

19. Gatski, T.B., Speziale, C,G,: On explicit algebraic stress models for complex turbulent flows. J. Fluid Mech. 254, 59-78 (1993)

20. Girimaji, S.S.: Fully explicit and self-consistent algebraic Reynolds stress model. Theor. Comput. Fluid Dyn. 8(6), 387-402 (1996)

21. Grad, H.: On the kinetic theory of rarefied gases. Commun. Pure Appl. Math. 2, 331-407 (1949)

22. Gustavsson, K., Meneguz, E., Reeks, M., Mehlig, B.: Inertial-particle dynamics in turbulent flows: caustics, concentration fluctuations, and random uncorrelated motion. New J. Phys. 14, 115017 (2012)

23. He, J., Simonin, O.: Non-equilibrium prediction of the particle-phase stress tensor in vertical pneumatic conveying. In: Proceedings of the 5th International Symposium on Gas-Solid Flows, vol. 166, pp. 253-263. ASME FED (1993)

24. IJzermans, R.H.A., Meneguz, E., Reeks, M.W.: Segregation of particles in incompressible random flows: singularities, intermittency and random uncorrelated motion. J. Fluid Mech. 653, 99-136 (2010)

25. Kah, D., Laurent, F., Fréret, L., de Chaisemartin, S., Fox, R.O., Reveillon, J., Massot, M.: Eulerian quadrature-based moment models for dilute polydisperse evaporating sprays. Flow Turbul. Combust. 85(3-4), 649-676 (2010)

26. Kaufmann, A., Moreau, M., Simonin, O., Helie, J.: Comparison between Lagrangian and mesoscopic Eulerian modelling approaches for inertial particles suspended in decaying isotropic turbulence. J. Comput. Phys. 227(13), 6448-6472 (2008)

27. Laurent, F., Massot, M.: Multi-fluid modeling of laminar poly-dispersed spray flames: origin, assumptions and comparison of sectional and sampling methods. Combust. Theor. Model. 5, 537-572 (2001)

28. Le Lostec, N., Villedieu, P., Simonin, O.: Quadrature-based third order moment models for the simulation of particle laden flows. In: Proceedings in the 7th International Conference on Multiphase Flow (ICMF). Tampa, Florida, USA, 30 May-4 June 2010

29. Marchisio, D.L., Fox, R.O.: Solution of population balance equations using the direct quadrature method of moments. J. Aerosol Sci. 36(1), 43-73 (2005)

30. Masi, E.: Theoretical and numerical study of the modeling of unsteady non-isothermal particleladen turbulent flows by an Eulerian-Eulerian approach. Ph.D. thesis, Institut National Polytechnique de Toulouse (2010) 
31. Masi, E., Bédat, B., Moreau, M., Simonin, O.: Euler-Euler large-eddy simulation approach for non isothermal particle-laden turbulent jet. In: Proceedings of the ASME - Fluids Engineering Division Summer Meeting, vol. 1, pp. 111-120. Symposia, Parts A and B, Paper No. FEDSM200855143. Jacksonville, Florida, USA, 10-14 August 2008

32. Masi, E., Riber, E., Sierra, P., Simonin, O., Gicquel, L.Y.M.: Modeling the random uncorrelated velocity stress tensor for unsteady particle Eulerian simulation in turbulent flows. In: Proceedings of the 7th International Conference on Multiphase Flow (ICMF). Tampa, Florida, 30 May-4 June 2010

33. Masi, E., Simonin, O., Bédat, B.: The mesoscopic Eulerian approach for evaporating droplets interacting with turbulent flows. Flow Turbul. Combust. 86, 563-583 (2011)

34. Massot, M., Laurent, F., Kah, D., de Chaisemartin, S.: A robust moment method for evaluation of the disappearance rate of evaporating sprays. SIAM J. Appl. Math. 70(8), 3203-3234 (2010)

35. Maxey, M.R.: The gravitational settling of particles in homogeneous turbulence and random flow. J. Fluid Mech. 26, 441 (1987)

36. Maxey, M.R., Riley, J.J.: Equation of motion for a small rigid sphere in a non uniform flow. Phys. Fluids 26, 2883-2889 (1983)

37. Meneguz, E., Reeks, M.W.: Statistical properties of particle segregation in homogeneous isotropic turbulence. J. Fluid Mech. 686, 338-351 (2011)

38. Moreau, M.: Modélisation numérique directe et des grandes échelles des écoulements turbulents gaz-particules dans le formalisme Eulérien mésoscopique. Ph.D. thesis, Institut National Polytechnique de Toulouse (2006)

39. Moreau, M., Simonin, O., Bédat, B.: Development of gas-particle Euler-Euler LES approach: a priori analysis of particle sub-grid models in homogeneous isotropic turbulence. Flow Turbul. Combust. 84(2), 295-324 (2010)

40. Passalacqua, A., Fox, R.O., Garg, R., Subramaniam, S.: A fully coupled quadrature-based moment method for dilute to moderately dilute fluid-particle flows. Chem. Eng. Sci. 65(7), 2267-2283 (2010)

41. Pope, S.B.: A more general effective-viscosity hypothesis. J. Fluid Mech. 72(02), 331-340 1975

42. Pope, S.B.: Turbulent Flows. Cambridge University Press, Cambridge, United Kingdom (2000)

43. Prevost, F., Boree, J., Nuglisch, H.J., Charnay, G.: Measurements of fluid/particle correlated motion in the far field of an axisymmetric jet. Int. J. Multiphase Flow 22(4), 685-701 (1996)

44. Rani, S.L., Balachandar, S.: Evaluation of the equilibrium Eulerian approach for the evolution of particle concentration in isotropic turbulence. Int. J. Multiphase Flow 29, 1793-1816 (2003)

45. Ranz, W.E., Marshall, W.R.: Evaporation from drops: I. Chem. Eng. Prog. 48, 141-146 (1952)

46. Reeks, M.W.: On a kinetic equation for the transport of particles in turbulent flows. Phys. Fluids 3(3), 446-456 (1991)

47. Riber, E.: Développement de la méthode de simulation aux grandes échelles pour les écoulements diphasiques turbulents. Ph.D. thesis, Institut National Polytechnique de Toulouse (2007)

48. Rotta, J.: Statistische theorie nichthomogener turbulenz. I. Z. Phys. 129, 547-572 (1951)

49. Sakiz, M., Simonin, O.: Numerical experiments and modelling of non-equilibrium effects in dilute granular flows. In: Brun, R., Campargue, R., Gatignol, R., Lengrand, J.C. (eds.) Rarefied Gas Dynamics, Proceedings of the 21st International Symposium on Rarefied Gas Dynamics, Cépaduès Editions, vol. 1, pp. 287-294. Marseille (France) (1999)

50. Schiller, L., Nauman, A.: A drag coefficient correlation. V.D.I. Zeitung 77, 318-320 (1935)

51. Shotorban, B., Balachandar, S.: Particle concentration in homogeneous shear turbulence simulated via Lagrangian and equilibrium Eulerian approaches. Phys. Fluids 18(6), 065105 (2006)

52. Sierra-Sánchez, P.: Modeling the dispersion and evaporation of sprays in aeronautical combustion chambers. Ph.D. thesis, Institut National Polytechnique de Toulouse (2012)

53. Simonin, O.: Second-moment prediction of dispersed phase turbulence in particle-laden flows. In: Proceedings of the 8th Symposium on Turbulent Shear Flows, vol. 1, pp. 741-746. Munich, Federal Republic of Germany, 9-11 September 1991

54. Simonin, O.: Prediction of the dispersed phase turbulence in particle-laden jets. In: Proceedings of the 4th International Symposium on Gas-Solid Flows, vol. 121, pp. 197-206. ASME FED (1991)

55. Simonin, O.: Second-moment prediction of the dispersed phase turbulence in particle-laden flows. Annexe 3: third-moment closure. In: Rapport EDF HE44/91.24 (1991)

56. Simonin, O., Février, P., Laviéville, J.: On the spatial distribution of heavy particle velocities in turbulent flow: from continuous field to particulate chaos. J. Turbul. 3, N40 (2002) 
57. So, R.M.C., Jin, L.H., Gatski, T.B.: An explicit algebraic Reynolds stress and heat flux model for incompressible turbulence: part I non-isothermal flow. Theor. Comput. Fluid Dyn. 17(5), 351-376 (2004)

58. Spencer, A.J.M.: Theory of Invariants. In: Eringen A.C. (ed.) Continuum Physics vol. I, part III. Academic Press, New York (1971)

59. Struchtrup, H., Torrilhon, M.: Regularization of Grad's 13 moment equations: derivation and linear analysis. Phys. Fluids 15, 2668-2680 (2003)

60. Torrilhon, M., Struchtrup, H.: Regularized 13-moment equations: shock structure calculations and comparison to Burnett models. J. Fluid Mech. 513, 171-198 (2004)

61. Vermorel, O., Bédat, B., Simonin, O., Poinsot, T.: Numerical study and modelling of turbulence modulation in a particle laden slab flow. J. Turbul. 4, N25 (2003)

62. Vié, A., Chalons, C., Fox, R.O., Laurent, F., Massot, M.: A multi-Gaussian quadrature method of moments for simulating high stokes number turbulent two-phase flows. In: Annual Research Briefs of the CTR, pp. 309-320 (2011)

63. Vié, A., Masi, E., Simonin, O., Massot, M.: On the direct numerical simulation of moderatestokes-number turbulent particulate flows using algebraic-closure-based and kinetic-based moment methods. In: Proceedings of the Center for Turbulence Research (CTR) Summer Program, pp. 355-364 (2012)

64. Wallin, S., Johansson, A.V.: An explicit algebraic Reynolds stress model for incompressible and compressible turbulent flows. J. Fluid Mech. 403, 89-132 (2000)

65. Wikström, P.M., Wallin, S., Johansson, A.V.: Derivation and investigation of a new explicit algebraic model for the passive scalar flux. Phys. Fluids 12(3), 688-702 (2000)

66. Wong, C.K., McLennan, J.A., Lindenfeld, M., Dufty, J.W.: Theory of nonlinear transport in Burnett order. J. Chem. Phys. 68, 1563-1573 (1978)

67. Yuan, C., Fox, R.O.: Conditional quadrature method of moments for kinetic equations. J. Comput. Phys. 230(22), 8216-8246 (2011)

68. Zaichik, L.I.: A statistical model of particle transport and heat transfer in turbulent shear flows. Phys. Fluids 11(6), 1521-1534 (1999)

69. Zaichik, L.I., Vinberg, A.A.: Modelling of particle dynamics and heat transfer in turbulent flows using equations for first and second moments of velocity and temperature fluctuations. In: Proceedings of the 8th Symposium on Turbulent Shear Flows, vol. 1, pp. 1021-1026. Munich, Federal Republic of Germany, 9-11 September 1991

70. Zhou, L.X.: Advances in studies on two-phase turbulence in dispersed multiphase flows. Int. J. Multiphase Flow 36(2), 100-108 (2010) 